J. DIFFERENTIAL GEOMETRY

48 (1998) 91-133

\title{
INTEGRAL INVARIANTS OF 3-MANIFOLDS
}

\author{
RAOUL BOTT \& ALBERTO S. CATTANEO
}

\begin{abstract}
This note describes an invariant of rational homology 3-spheres in terms of configuration space integrals, which in some sense lies between the invariants of Axelrod and Singer [2] and those of Kontsevich [9].
\end{abstract}

\section{Introduction}

In their seminal paper [2] of 1994, Axelrod and Singer showed that the asymptotics of the Chern-Simons theory led to a series of $C^{\infty}$ invariants associated to triples $\{M ; f ; \rho\}$ with $M$ a smooth homology 3-sphere, $f$ a homotopy class of framings of $M$, and $\rho$ an "acyclic" conjugacy class of orthogonal representations of $\pi_{1}(M)$. That is, the cohomology $H^{*}(M ; \operatorname{Ad} \rho)$ of $M$ relative to the local system associated to $\operatorname{Ad} \rho$ vanishes.

The primary purpose of this note is to show that the basic ideas of their paper can be adapted quite easily - but not quite trivially - to yield invariants of smooth, framed 3-dimensional homology spheres as such. Put differently, we will present a treatment somewhat analogous to theirs for the trivial representation of $\pi_{1}(M)$. We say somewhat because in our work we have put aside the physics inspired aspects of Axelrod and Singer's paper. Instead we have simply taken our task to be the production of invariants of framed manifolds $(M ; f)$ out of some fixed Riemannian structure on $M$.

There is of course Kontsevich's solution by "softer methods" to the problem of finding the residual invariants of the Chern-Simons theory

Received July 2, 1997. We thankfully acknowledge support from NSF for R.B. and from INFN (grant No. 5565/95) and from DOE (grant No. DE-FG02-94ER25228, Amendment No. A003) for A.S.C. 
at the trivial representation. In a note (see [9]), Kontsevich sketched how to define a series of invariants for "framed" 3-dimensional homology spheres, and developed his "graph cohomology" to explain the combinatorial diversity of these invariants.

In 1995 Taubes [10] carefully investigated the first of these Kontsevich invariants - the one associated to the $\Theta$-graph-and clarified the appropriate concept of "framing" for all of the graph cohomology. He coined the term "singular framings" for them, and they differ from ordinary framings in that they exhibit a singularity at one point of $M$.

Our invariants - which are less "soft" than Kontsevich's in the sense that they do depend on Riemannian concepts - are therefore, on the face of it, different from his. But they are also indexed by cocycles, $\Gamma$, in an appropriate graph cohomology, and structurally take the form:

$$
I_{\Gamma}(M, f)=A_{\Gamma}(M)+\phi(\Gamma) \operatorname{CS}(M, f) .
$$

Here $A_{\Gamma}(M)$ is an integral over the configuration spaces specified by $\Gamma$ and defined by a fixed Riemannian structure on $M, \phi(\Gamma)$ is a real number universally associated to $\Gamma$, and $\operatorname{CS}(M, f)$ denotes the ChernSimons integral of the Levi-Civita connection of $M$ relative to the frame $f$.

The Axelrod-Singer invariants for a flat connection exhibit a similar dependance on the framings, and (1.1) is also in general conformity with the self-linking invariants in knot theory - as described in [5] (see also [1]). There the invariants of a knot $K \subset \mathbb{R}^{3}$ are described as

$$
I_{\Gamma}(K)=A_{\Gamma}(K)+\mu(\Gamma) \cdot \text { self-linking of } K,
$$

where $I_{\Gamma}(K)$ is a configuration space integral which is corrected by an anomalous term which is a multiple of the self-linking of $K$.

Similarly we now obtain invariants of oriented homology 3 -spheres, one for every connected cocycle $\Gamma$, of the form

$$
J_{\Gamma}(M)=A_{\Gamma}(M)-4 \phi(\Gamma) A_{\Theta}(M),
$$

so that $A_{\Theta}(M)$ is seen to play the role of the self-linking integral in knot theory.

Although the invariants of [2] and [5], as well as the ones described here, are all spin-offs from Witten's [11] original Chern-Simons invariants for homology 3 -spheres, it seems to us that, from a purely mathematical point of view, they have now, in retrospect, even older antecedents. These are the "iterated integrals" of Chern, or-even olderthe Adams constructions for the loop-space of a space. 
Quite generally, the principle of these constructions is to describe the cohomology of a function-space $F=\operatorname{Map}(X, Y)$ in terms of the various evaluation maps:

$$
\operatorname{Map}(X, Y) \times X^{n} \rightarrow Y^{n}
$$

When we are dealing with corresponding spaces of imbeddings, or diffeomorphisms, then the configuration spaces enter the discussion quite naturally, and give rise to new invariants of the type we have been discussing.

In this context it is also possible to extend our considerations to local systems on $M$, and derive similar invariants, all governed by some graph cohomology. From this point of view the original invariants of AxelrodSinger are associated to the "Feynman cocycles" of this cohomology.

We hope to explore these ideas in a subsequent paper [4]. Here we will only deal with the constant coefficient case and the plan of this note is as follows: In section 2 we review some facts from the theory of characteristic classes. In section 3 we describe the $\Theta$-invariant explicitly, but implicitly rely on the description of configuration spaces as developed in [2], in analogy with the corresponding algebraic construction given by Fulton and MacPherson in [7]. In section 4 we discuss the higher invariants while the last, fifth, section is devoted to extending the results of [5] to knots in general homology 3 -spheres.

\section{Acknowledgements}

We are indebted for very useful conversations pertaining to these matters with Scott Axelrod, Robin Forman, Stavros Garoufalidis and Cliff Taubes.

\section{Review of characteristic classes of $S O(n)$}

Consider an oriented vector bundle $E$ with odd fiber dimension, $n=2 k+1$, over a base space $M$. Also let $S(E)$ denote the associated sphere bundle to $E$, which we may consider to be the space of rays in $E$; or, if $E$ is given a Riemannian structure, as the unit sphere bundle of $E$. In any case $S(E)$ has even fiber dimension $2 k$ over $M$, and this together with the orientability of $E$ allows one to specify a canonical integral generator of the rational cohomology of $S(E)$ as a module over $H^{*}(M)$. Namely, we consider the "tangent bundle along the fiber," $T_{F} S(E)$, of 
$S(E)$. This, being an even dimensional oriented bundle, has a canonical Euler class:

$$
e=e\left(T_{F} S(E)\right) \in H^{2 k}(S(E))
$$

which restricts to twice the generator of $H^{2 k}\left(S^{2 k}\right)$ on each fiber, because the Euler number of $S^{2 k}$ is 2 .

But then it follows from general principles that $e$ generates $H^{*}(S(E))$ over $H^{*}(M)$ over the rationals.

Concerning the generator $e$ we have the following lemma, which in some sense explains the Chern-Simons term in our subsequent construction.

Lemma 2.1. Let $\pi_{*}$ denote integration along the fiber in the bundle $S(E)$ over $M$. Then

$$
\pi_{*} e^{3}=2 p_{k}(E),
$$

where $p_{k}$ denotes the $k$-th Pontrjagin class of $E$. In fact one has, quite generally:

$$
\pi_{*} e^{2 s+1}=2\left(p_{k}(E)\right)^{s},
$$

and

$$
\pi_{*} e^{2 s}=0
$$

for $s=1,2, \ldots$.

Proof. It suffices to prove these formulae for the universal sphere bundle over the classifying space $B S O(n), n=2 k+1$, that is for the fibering

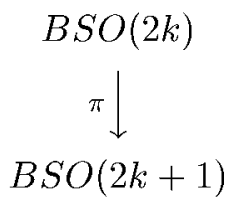

with fiber $S O(2 k+1) / S O(2 k)=S^{2 k}$.

Here we can keep track of the rational cohomology of the spaces involved by choosing a maximal torus $T=\left(S^{1}\right)^{k}$ for $S O(2 k+1)$ in the usual manner, so that $T$ corresponds to diagonal $2 \times 2$ blocks ending with a 1 in the last diagonal position. In this way $H^{*}(B T)$ becomes 
identified with the polynomial ring $\mathbb{Q}\left[x_{1}, \ldots, x_{k}\right]$ and the Weyl group of $S O(2 k+1)$ acts on this cohomology by 1$)$ permutations of the $x_{i}$, and 2) changes of sign $x_{i} \rightarrow \pm x_{i}$. On the other hand the Weyl group of $S O(2 k)$ acts as the subgroup which 1 ) permutes the $x_{i}$, and 2) allows only even changes of $\operatorname{sign} x_{i} \rightarrow \epsilon_{i} x_{i}, \epsilon_{i}= \pm 1$, with $\prod \epsilon_{i}=1$.

It follows that the invariants of $H^{*}(B T)$ under $S O(2 k+1)$ are given by the invariant polynomials $\sigma_{r}=\sigma_{r}\left(x_{1}^{2}, \ldots, x_{k}^{2}\right), r \leq k$, while those invariant under $S O(2 k)$ are generated by $\sigma_{r}$ and an additional element

$$
e=x_{1} \ldots x_{k} \in H^{2 k}(B T) .
$$

From the well-known identification of $H^{*}(B S O(2 k+1))$ and $H^{*}(B S O(2 k))$ with these rings of invariants respectively, we conclude that:

$$
e^{2}=\pi^{*}\left(x_{1}^{2} \ldots x_{k}^{2}\right)=\pi^{*} \sigma_{k} \quad \text { in }(2.6)
$$

But then

$$
\pi_{*} e^{3}=\pi_{*}\left(e \pi^{*} \sigma_{k}\right)=2 \sigma_{k}
$$

by the permanence relation and the fact that $\pi_{*} e=2$ remarked upon earlier. If we take $\sigma_{r}$ to be universal Pontrjagin class-as opposed to the convention $p_{r}=(-1)^{r} \sigma_{r}$-then (2.7) implies (2.2), and the general case follows similarly from $e^{2 s+1}=e \pi^{*}\left(\sigma_{k}\right)^{s}$. q.e.d.

\section{The simplest invariant}

From now on we will only consider a 3 -dimensional rational homology sphere $M$. The boundary of the configuration space of two points in $M, C_{2}(M)$, is then isomorphic to the 2-sphere bundle $S(T M)$ over $M$. In the previous section we have seen how to construct a vertical generator $e$ in a sphere bundle using Riemannian concepts. In this section we will give this generator explicitly as an element of $\Omega^{2}\left(\partial C_{2}(M)\right)$. In the de Rham theory we can divide by 2 and so we will actually describe $\eta=e / 2$. Then we will extend it to the whole of $\Omega^{2}\left(C_{2}(M)\right)$ and will show that its differential is the Poincaré dual of the diagonal in $M \times M$. The next step will be to use this element to construct a closed form in $\Omega^{2}\left(C_{3}(M)\right)$. It is precisely through this form that we will be able to write the simplest invariant of the rational homology sphere $M$ as an integral over $C_{3}(M)$ (notice that in [2], [9] and [10] the "corresponding" 
$\Theta$-invariant is written as an integral over $\left.C_{2}(M)\right)$. Finally, we will prove that, apart from an anomalous term which we compute explicitly, this is actually an invariant.

3.1. The generator of $H^{2}\left(\partial C_{2}(M)\right)$. We may consider $\partial C_{2}(M)$ as the sphere bundle $P \times_{S O(3)} S^{2} \rightarrow M$ where $P \rightarrow M$ is the orthonormal frame bundle of $T M$ with respect to some fixed Riemannian structure, so that we have the commutative diagram:

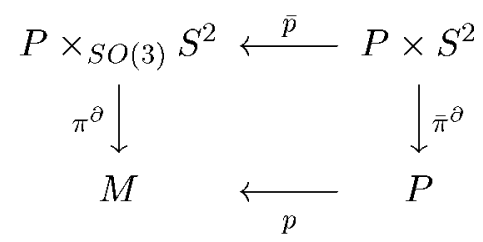

Note that here $\bar{\pi}^{\partial}$ is a morphism of principal $S O(3)$-bundles while $\bar{p}$ is a corresponding morphism of $S^{2}$-bundles.

We will write our class $\eta$, or more precisely $\bar{p}^{*} \eta$, as a closed form in $\Omega_{\text {basic }}^{2}\left(P \times S^{2}\right)$ such that $\bar{\pi}_{*}^{\partial} \bar{p}^{*} \eta=1$. For the $(0,2)$ component of $\bar{p}^{*} \eta$ we choose the $S O(3)$-invariant volume element

$$
\omega=x d y d z+y d z d x+z d x d y=\frac{1}{2} \epsilon^{i j k} x_{i} d x_{j} d x_{k},
$$

which satisfies $\int_{S^{2}} \omega=4 \pi$. The $S O(3)$-action on $S^{2}$ is given by the vector fields

$$
X_{i}=\epsilon_{i j}{ }^{k} x_{j} \frac{\partial}{\partial x_{k}} .
$$

We have

$$
L_{X_{i}} \omega=0, \quad \iota_{X_{i}} \omega=d x_{i} .
$$

Let $\left\{\xi_{1}, \xi_{2}, \xi_{3}\right\}$ be the basis of $\mathfrak{s o}(3)$ that corresponds to the vector fields $X_{1}, X_{2}, X_{3}$; that is,

$$
\left(\xi_{i}\right)_{j k}=\epsilon_{i j k} .
$$

Then a connection $\theta$ on $P$ can be expanded in this basis as

$$
\theta=\theta^{i} \xi_{i}
$$

so that, by the definition of a connection, we have

$$
L_{X_{i}} \theta^{j}=-\epsilon_{i j k} \theta^{k}, \quad \iota_{X_{i}} \theta^{j}=\delta_{i}^{j} .
$$


In the following we will write $\theta^{i}$ also for the pullback of $\theta^{i}$ to $P \times S^{2}$, and similarly consider the coordinates $x_{i}$ of $\mathbb{R}^{3}$ also as pulled back to $P \times S^{2}$. This understood consider the invariant 1 -form $\theta^{i} x_{i}$. It then follows from this invariance that

$$
\iota_{X_{i}} d\left(\theta^{j} x_{j}\right)=-d\left(\iota_{X_{i}} \theta^{j} x_{j}\right)=-d x_{i} .
$$

Next, we have the following

Proposition 3.1. The 2-form

$$
\bar{\eta}=\frac{\omega+d\left(\theta^{i} x_{i}\right)}{4 \pi}, \quad x^{i} x_{i}=1
$$

is basic in $P \times S^{2} \rightarrow P \times_{S O(3)} S^{2}$. Moreover, if we write $\bar{\eta}=\bar{p}^{*} \eta$, then

$$
\pi_{*}^{\partial} \eta=1, \quad \pi_{*}^{\partial} \eta^{2}=0
$$

Finally, if $\phi$ is the automorphism of the bundle $P \times_{S O(3)} S^{2} \rightarrow M$ given by the antipodal map on the fiber, then

$$
\phi^{*} \eta=-\eta .
$$

Proof. Eqn. (3.1) follows directly from the previous discussion.

To prove (3.2), we notice that $p^{*} \pi_{*}^{\partial}=\bar{\pi}_{*}^{\partial} \bar{p}^{*}$. Thus, the first identity is just a consequence of the fact that

$$
\bar{\pi}_{*}^{\partial}\left[\omega+d\left(\theta^{i} x_{i}\right)\right]=\bar{\pi}_{*}^{\partial} \omega=4 \pi .
$$

For the second identity we compute

$$
\begin{aligned}
(4 \pi)^{2} \bar{\pi}_{*}^{\partial}\left(\bar{p}^{*} \eta\right)^{2} & =\bar{\pi}_{*}^{\partial}\left[2 \omega d\left(\theta^{i} x_{i}\right)+d\left(\theta^{i} x_{i}\right) d\left(\theta^{j} x_{j}\right)\right] \\
& =2 d \theta^{i} \bar{\pi}_{*}^{\partial}\left(\omega x_{i}\right)-\theta^{i} \theta^{j} \bar{\pi}_{*}^{\partial}\left(d x_{i} d x_{j}\right)=0 .
\end{aligned}
$$

For the last identity, notice that the integral of $\omega x_{i}$ vanishes by symmetry and that $d x_{i} d x_{j}$ is exact.

Finally, to prove (3.3), we consider the automorphism $\bar{\phi}$ of $P \times S^{2} \rightarrow$ $P$ obtained by the antipodal map on $S^{2}$ :

$$
\bar{\phi} x_{i}=-x_{i}
$$

Clearly, $\bar{\phi}^{*} \bar{p}^{*} \eta=-\bar{p}^{*} \eta$. Moreover, $\phi \bar{p}=\bar{p} \bar{\phi}$. Then

$$
\bar{p}^{*} \phi^{*} \eta=\bar{\phi}^{*} \bar{p}^{*} \eta=-\bar{p}^{*} \eta
$$

which implies (3.3). q.e.d. 
Digression. If we introduce the covariant derivative $D$,

$$
(D x)_{i}=d x_{i}-\epsilon_{i j}{ }^{k} \theta^{j} x_{k}
$$

and the curvature $F=F^{i} \xi_{i}$,

$$
F^{i}=d \theta^{i}-\frac{1}{2} \epsilon^{i}{ }_{j k} \theta^{j} \theta^{k},
$$

then a straightforward but tedious computation shows that we can also write

$$
\bar{p}^{*} \eta=\frac{\frac{1}{2} \epsilon^{i j k} x_{i}(D x)_{j}(D x)_{k}+F^{i} x_{i}}{4 \pi} .
$$

In fact,

$$
\begin{aligned}
\frac{1}{2} \epsilon^{i j k} x_{i}(D x)_{j}(D x)_{k}= & \frac{1}{2} \epsilon^{i j k} x_{i} d x_{j} d x_{k}-\frac{1}{2} \epsilon^{i j k} x_{i} \epsilon_{j r}{ }^{s} \theta^{r} x_{s} d x_{k} \\
& -\frac{1}{2} \epsilon^{i j k} x_{i} d x_{j} \epsilon_{k r}{ }^{s} \theta^{r} x_{s} \\
& +\frac{1}{2} \epsilon^{i j k} x_{i} \epsilon_{j r}{ }^{s} \theta^{r} x_{s} \epsilon_{k m}{ }^{n} \theta^{m} x_{n} \\
= & \omega-\theta^{i} d x_{i}+\frac{1}{2} \epsilon^{i}{ }_{j k} x_{i} \theta^{j} \theta^{k} .
\end{aligned}
$$

To obtain the last identity we have used

$$
\sum_{i} \epsilon_{i j k} \epsilon_{i r s}=\delta_{j r} \delta_{k s}-\delta_{j s} \delta_{k r}
$$

and the constraint $x^{i} x_{i}=1$.

Note also that, if one considers $\mathbf{x}=x^{i} \xi_{i}$ as an element of the fundamental representation of $\mathfrak{s o}(3)$, then, by using the identities

$$
\operatorname{Tr} \xi_{i} \xi_{j}=-2 \delta_{i j}, \quad \operatorname{Tr} \xi_{i}\left[\xi_{j}, \xi_{k}\right]=2 \epsilon_{i j k},
$$

one can rewrite $(3.1)$ and $\left(? ?^{\prime}\right)$ as

$$
\bar{p}^{*} \eta=\frac{\omega-\frac{1}{2} d \operatorname{Tr}(\theta \mathbf{x})}{4 \pi}=\frac{\operatorname{Tr}(\mathbf{x} D \mathbf{x} D \mathbf{x}-F \mathbf{x})}{8 \pi} .
$$

End of the digression. 
3.2. The extension to $C_{2}(M)$. First we want to extend our form $\eta$ to a small neighborhood $U$ of $\partial C_{2}(M)$. We can think of this neighborhood as the complement $T M^{\prime}$ of the zero section of the tangent bundle $T M$. We still have an $S O(3)$-bundle

$$
P \times(\mathbb{R} \backslash 0) \stackrel{\bar{p}}{\rightarrow} T M^{\prime}
$$

Scaling each coordinate $x_{i}$ in $\mathbb{R} \backslash 0$ by $r=\left(x_{1}\right)^{2}+\left(x_{2}\right)^{2}+\left(x_{3}\right)^{2}$, we get the closed, basic form

$$
\bar{p}^{*} \eta=\frac{\omega}{4 \pi r^{3}}+\frac{1}{4 \pi} d\left(\frac{\theta^{i} x_{i}}{r}\right)
$$

with $\omega$ defined as before. Then we consider a second neighborhood $V$ containing $U$ and contained in $C_{2}(M)$, and choose a function $\rho$ on $C_{2}(M)$ that is constant and equal to -1 in $U$, and constant and equal to 0 in the complement of $V$. It is thus clear that $d(\rho \eta)$ represents a class in $H^{3}(M \times M)=H^{3}(M) \otimes H^{3}(M)$.

Let us denote by $\pi_{1}$ and $\pi_{2}$ the two natural projections from $M \times M$ to $M$, i.e.,

$$
\pi_{i}\left(m_{1}, m_{2}\right)=m_{i}
$$

and by $v$ a unit volume form on $M$ (not necessarily the volume form determined by the metric). In fact any $v$ with $\int_{M} v=1$ would do, and we will use the term "unit volume" form in this sense throughout. Then the generators of $H^{3}(M \times M)$ are $v_{1}$ and $v_{2}$, defined by

$$
v_{i}=\pi_{i}^{*} v
$$

and we can write $[d(\rho \eta)]=c_{1} v_{1}+c_{2} v_{2}$ for some constants $c_{1}$ and $c_{2}$. Since

$$
\int_{C_{2}(M)} d(\rho \eta) v_{i}=\int_{\partial C_{2}(M)} \rho \eta v_{i}=-\int_{\partial C_{2}(M)} \eta v_{i}=-\int_{M} v=-1,
$$

we see that actually

$$
[d(\rho \eta)]=v_{2}-v_{1} ;
$$

that is, $d(\rho \eta)$ represents the Poincare dual of the diagonal in $M \times M$. This means that there exists a form $\alpha \in \Omega^{2}(M \times M)$ such that

$$
d(\rho \eta)=v_{2}-v_{1}-d \alpha .
$$


Now consider the involution

$$
\begin{aligned}
T: C_{2}(M) & \rightarrow C_{2}(M) \\
\left(m_{1}, m_{2}\right) & \mapsto\left(m_{2}, m_{1}\right),
\end{aligned}
$$

and its analog on $M \times M$, which we still denote by $T$. Since $T$ restricted to $\partial C_{2}(M)$ is the automorphism $\phi$ considered in Prop. 3.1, then

$$
T^{*}(\rho \eta)=-\rho \eta,
$$

provided we choose $\rho$ symmetric (e.g., we can take $\rho$ to be a function of the distance between $m_{1}$ and $m_{2}$ ). It follows that in (3.7) we can choose $\alpha$ such that

$$
T^{*} \alpha=-\alpha
$$

Define

$$
\hat{\eta}=\rho \eta+\alpha \in \Omega^{2}\left(C_{2}(M)\right)
$$

We have therefore proved

Proposition 3.2. There exist forms $\hat{\eta} \in \Omega^{2}\left(C_{2}(M)\right)$ with the following three properties:

$$
\begin{gathered}
\pi_{*}^{\partial} \iota_{\partial}^{*} \hat{\eta}=-1, \\
d \hat{\eta}=v_{2}-v_{1}, \\
T^{*} \hat{\eta}=-\hat{\eta} .
\end{gathered}
$$

Moreover, there exist forms $\hat{\eta}$ with the additional property

$$
\iota_{\partial}^{*} \hat{\eta}=-\eta .
$$

Here $\iota_{\partial}$ is the inclusion $\partial C_{2}(M) \hookrightarrow C_{2}(M)$.

Remark 3.3. A metric, a compatible connection and a unit volume form are not enough to determine a unique $\hat{\eta}$, for

$$
\hat{\eta}^{\prime}=\hat{\eta}+d \beta
$$

with $\beta \in \Omega^{1}\left(C_{2}(M)\right)$ such that $T^{*} \beta=-\beta$, still satisfies (3.10). If we moreover want $\hat{\eta}$ to satisfy $(3.11)$, then we must also put the restriction that $\iota_{\partial}^{*} \beta=0$. 
Digression (The Riemannian parametrix). Given a Riemannian structure $g$ on a manifold $M$, a linear operator

$$
P_{g}: \Omega^{*}(M) \rightarrow \Omega^{*-1}(M)
$$

with the property that

$$
d P_{g}+P_{g} d=1-\pi_{h},
$$

where $\pi_{h}$ is the orthogonal projection onto the harmonic forms, will be called a Riemannian parametrix. Of course (3.13) does not define a unique $P_{g}$, for

$$
P_{g}^{\prime}=P_{g}+d Q-Q d
$$

still satisfies it for any $Q: \Omega^{*}(M) \rightarrow \Omega^{*-2}(M)$.

The harmonic projection can be written as a convolution on $M \times M$ (or $C_{2}(M)$ ) as

$$
\pi_{h} \alpha=\pi_{2 *}\left(\eta_{\Delta} \pi_{1}^{*} \alpha\right)
$$

where $\eta_{\Delta}$ is the representative of the Poincaré dual of the diagonal in $M \times M$ determined by the metric on $M$. In the case where $M$ is a rational homology sphere we have $\eta_{\Delta}=v_{2}-v_{1}$.

Now we have the following proposition.

Proposition 3.4. A form $\hat{\eta} \in \Omega^{2}\left(C_{2}(M)\right)$ satisfying (3.10) with $v$ the volume form determined by the metric $g$ is the Schwartz kernel for a Riemannian parametrix $P_{g}$. More precisely, given a form $\alpha \in \Omega^{*}(M)$, the operator $P_{g}$ defined by

$$
P_{g} \alpha=-\pi_{2 *}\left(\hat{\eta} \pi_{1}^{*} \alpha\right)
$$

satisfies (3.13).

Proof. We need the following generalization of Stokes' formula:

$$
d \pi_{2 *}=-\pi_{2 *} d+\pi_{*}^{\partial} \iota_{\partial}^{*},
$$

which holds in the case of an odd-dimensional fiber with boundary. It follows that

$$
\begin{aligned}
d P_{g} \alpha+P_{g} d \alpha & =\pi_{2 *}\left(d \hat{\eta} \pi_{1}^{*} \alpha\right)-\pi_{*}^{\partial} \iota_{\partial}^{*}\left(\hat{\eta} \pi_{1}^{*} \alpha\right) \\
& =-v \pi_{2 *} \pi_{1}^{*} \alpha-\pi_{2 *} \pi_{1}^{*}(v \alpha)+\alpha \pi_{*}^{\partial} \eta=\left(1-\pi_{h}\right) \alpha,
\end{aligned}
$$

where we have also used the fact that $\iota_{\partial}^{*} \pi_{i}^{*}=\pi^{\partial *}$. q.e.d. 
Remark 3.5. To define the Riemannian parametrix, we have only used properties (3.10). The additional property (3.11) will be crucial to define the manifold invariants. Notice, moreover, that the freedom (3.12) in defining $\hat{\eta}$ corresponds to the freedom (3.14) in defining $P_{g}$.

Remark 3.6. A particular choice of $P_{g}$ is given by $\bar{P}_{g}=d^{*} \circ G$, where $G$ is the inverse of $\square+\pi_{h}$ and $\square$ is the Laplace operator determined by $g$. We will not concentrate our interest on this particular Riemannian parametrix - as was the case in [2] — but will stick to the general case. In [2] it is precisely the Schwartz kernel for this Riemannian parametrix $\bar{P}_{g}$ that is constructed, and found to be represented on the boundary precisely by the form $\eta$ we have been considering. Close to the boundary there are corrections which are continuous but not smooth as forms on $M \times M$ (corresponding to the singular part of $G$ ). These forms, however, become smooth when lifted to $C_{2}(M)$. Then, with a suitable choice of $\beta \in \Omega^{1}\left(C_{2}(M)\right)$ in (3.12), we can recover the $\hat{\eta}$ representing $\bar{P}_{g}$.

End of the digression.

3.3. Extension to $C_{3}(M)$. Consider the three natural projections $\pi_{1}, \pi_{2}$ and $\pi_{3}$ from $C_{3}(M)$ to $M$ given by

$$
\pi_{i}\left(m_{1}, m_{2}, m_{3}\right)=m_{i}
$$

and call

$$
v_{i}=\pi_{i}^{*} v .
$$

Then consider the three natural projections $\pi_{12}, \pi_{23}$ and $\pi_{13}$ from $C_{3}(M)$ to $C_{2}(M)$ :

$$
\pi_{i j}\left(m_{1}, m_{2}, m_{3}\right)=\left(m_{i}, m_{j}\right), \quad 1 \leq i<j \leq 3
$$

and define

$$
\pi_{j i}=T \pi_{i j}
$$

where $T$ is the involution defined in (3.8). We will denote by

$$
\hat{\eta}_{i j}=\pi_{i j}^{*} \hat{\eta}
$$

the pullbacks of the form $\hat{\eta}$ defined in (3.9). We can recast the properties of $\hat{\eta}$ as

$$
\begin{aligned}
d \hat{\eta}_{i j} & =v_{j}-v_{i}, \\
\hat{\eta}_{j i} & =-\hat{\eta}_{i j} .
\end{aligned}
$$


Finally, introduce

$$
\hat{\eta}_{i j k}=\hat{\eta}_{i j}+\hat{\eta}_{j k}+\hat{\eta}_{k i},
$$

for $i, j$ and $k$ different from each other. A simple consequence of (3.17) is:

$$
\begin{aligned}
d \hat{\eta}_{i j k} & =0, \\
\hat{\eta}_{i j k} & =\epsilon_{i j k} \hat{\eta}_{123} .
\end{aligned}
$$

This way we have constructed a closed form in $\Omega^{2}\left(C_{3}(M)\right)$.

Remark 3.7. The form $\hat{\eta}$ depends on the choice of the unit volume form. In fact, if we pick up a different volume form $v^{\prime}=v+d \tau$, then, by (3.10b), we must replace $\hat{\eta}_{i j}$ by $\hat{\eta}_{i j}^{\prime}=\hat{\eta}_{i j}+\tau_{j}-\tau_{i}$. From (3.18), we see that $\hat{\eta}_{i j k}$ is unchanged.

We have not used property (3.11) yet. First notice that the boundary of $C_{3}(M)$ has four faces of codimension one, which we denote by (12), (23), (31) and (123), by indicating the underlying diagonal. Then it follows that

$$
\begin{aligned}
& \iota_{(12)}^{*} \hat{\eta}_{123}=-\eta_{12}, \\
& \iota_{(23)}^{*} \hat{\eta}_{123}=-\eta_{23}, \\
& \iota_{(31)}^{*} \hat{\eta}_{123}=-\eta_{31},
\end{aligned}
$$

and

$$
\iota_{(123)}^{*} \hat{\eta}_{123}=-\left(\eta_{12}+\eta_{23}+\eta_{31}\right) .
$$

Here by $\eta_{i j}$ we mean the pullback of the form $\eta \in \partial C_{2}(M)$ by the restriction to the boundary of the maps $\pi_{i j}$.

More precisely, a face like (12) is a sphere bundle over $C_{2}(M)$. If we denote by $m_{1}$ the point in $C_{2}(M)$ where the collapse has happened, then (12) can be expressed as $\pi_{1}^{-1} \partial C_{2}(M)$, where $\pi_{1}$ is the corresponding projection $C_{2}(M) \rightarrow M$. Then $\eta_{12}=\pi_{1}^{*} \eta$. Similarly for the faces (23) and (31).

The face (123) is a bundle over $M$ whose fiber $F$ is given by $C_{3}\left(\mathbb{R}^{3}\right)$ modulo global translations and scalings. If we denote by $\mathbf{x}_{1}, \mathbf{x}_{2}$ and $\mathbf{x}_{3}$ the coordinates of $F$, then we have the projections

$$
\begin{aligned}
& \pi_{i j}: \quad F \quad \rightarrow \quad S^{2}, \quad i \neq j, \\
& \left(\mathbf{x}_{1}, \mathbf{x}_{2}, \mathbf{x}_{3}\right) \mapsto \frac{\mathbf{x}_{j}-\mathbf{x}_{i}}{\left|\mathbf{x}_{j}-\mathbf{x}_{i}\right|}
\end{aligned}
$$


and their trivial extension to $P \times F \rightarrow P \times S^{2}$. Since they are equivariant, they descend to $P \times_{S O(3)} F \rightarrow P \times_{S O(3)} \times S^{2}=\partial C_{2}(M)$. Then $\eta_{i j}=$ $\pi_{i j}^{*} \eta$.

Remark 3.8. The form $\hat{\eta}$ is defined up to the differential of a 1form that vanishes on the boundary. Under the transformation (3.12), we have $\hat{\eta}_{123}^{\prime}=\hat{\eta}_{123}+d\left(\beta_{12}+\beta_{23}+\beta_{31}\right)$. More generally, since the properties we are interested in are (3.19) and (3.20), we can allow the addition of any exact term,

$$
\hat{\eta}_{123}^{\prime}=\hat{\eta}_{123}+d \beta,
$$

with $\beta \in \Omega^{1}\left(C_{3}(M)\right)$ and vanishing on the boundary.

3.4. The simplest invariant. We now have all the necessary elements to define the configuration space integral:

$$
A_{\Theta} \doteq \int_{C_{3}(M)} \hat{\eta}_{123}^{3} v_{1}
$$

The apparent asymmetry in the choice of $v_{1}$ can be removed if we notice that, by cyclically exchanging the three points in $C_{3}(M)$, we also have

$$
A_{\Theta}(M)=\int_{C_{3}(M)} \hat{\eta}_{123}^{3} v_{2}=\int_{C_{3}(M)} \hat{\eta}_{123}^{3} v_{3}=\frac{1}{3} \int_{C_{3}(M)} \hat{\eta}_{123}^{3}\left(v_{1}+v_{2}+v_{3}\right) .
$$

The definition of $A_{\Theta}$ relies on many choices: a metric, a connection compatible with that metric and a unit volume form; moreover, $\hat{\eta}_{123}$ is defined up to the differential of a 1-form that vanishes on the boundary. The last freedom is immediately seen not to have consequences on $A_{\Theta}$ since $\hat{\eta}_{123}$ and $v_{3}$ are closed forms. As we will see in the next subsection, $A_{\Theta}$ is not completely independent of all the other choices. However, we will be able to prove the following.

Theorem 3.9. Given a section $f$ of the frame bundle $P$, the combination

$$
I_{\Theta}(M, f)=A_{\Theta}(M)+\frac{1}{4} \operatorname{CS}(M, f)
$$

is independent of all the choices involved (except for the framing). Here

$$
\begin{aligned}
\operatorname{CS}(M, f) & =-\frac{1}{8 \pi^{2}} \int_{M} f^{*} \operatorname{Tr}\left(\theta d \theta+\frac{2}{3} \theta^{3}\right) \\
& =\frac{1}{4 \pi^{2}} \int_{M} f^{*}\left(\theta^{i} d \theta_{i}-\frac{1}{3} \epsilon_{i j k} \theta^{i} \theta^{j} \theta^{k}\right)
\end{aligned}
$$


is the Chern-Simons integral of the same metric connection used to define $\eta$.

Thus, $I_{\Theta}(M, f)$ is an invariant for the framed rational homology sphere $(M, f)$.

Remark 3.10. In an $S O(3)$-bundle, it is half the Chern-Simons form that restricted to the fiber yields the integral generator [6]. Therefore, the Chern-Simons term is defined up to an even integer, and the $\Theta$-invariant $I_{\Theta}$ up to half an integer.

Remark 3.11. So far we have considered $v$ to be a unit volume form (not necessarily determined by the metric). We can drop this assumption defining the invariant as

$$
I_{\Theta}(M, f)=\frac{1}{V^{4}} A_{\Theta}(M)+\frac{1}{4} \operatorname{CS}(M, f),
$$

where $V=\int_{M} v$. Notice that, for $\hat{\eta}$ to satisfy (3.10), we must now take the function $\rho$ in (3.9) to be constant and equal to $-V$ close to the boundary.

If one expands $\hat{\eta}_{123}^{3}$ in terms of the $\hat{\eta}_{i j}$ 's, one obtains $A_{\Theta}$ as the sum of nine integrals. However, many of these integrals vanish for purely dimensional reasons. After rearranging the points in $C_{3}(M)$, we can rewrite $A_{\Theta}$ as the sum of three contributions:

$$
A_{\Theta}(M)=A_{1}(M)+6 A_{2}(M)+6 A_{3}(M)
$$

with

$$
\begin{aligned}
& A_{1}(M)=\int_{C_{3}(M)} \hat{\eta}_{12}^{3} v_{3}=\int_{C_{2}(M)} \hat{\eta}^{3}, \\
& A_{2}(M)=\int_{C_{3}(M)} \hat{\eta}_{12}^{2} \hat{\eta}_{23} v_{3}, \\
& A_{3}(M)=\int_{C_{3}(M)} \hat{\eta}_{12} \hat{\eta}_{23} \hat{\eta}_{31} v_{3},
\end{aligned}
$$

which are graphically represented in Figure 1.

Remark 3.12. The integral $A_{1}(M)$ has the same form as the $\Theta-$ invariant in [2], [9] and [10].

Digression. The three integrals $A_{i}$ are not the only possible combinations containing three $\hat{\eta}$ 's. In fact we can also consider

$$
A_{4}(M)=\int_{C_{4}(M)} v_{1} \hat{\eta}_{12} \hat{\eta}_{23} \hat{\eta}_{34} v_{4}
$$




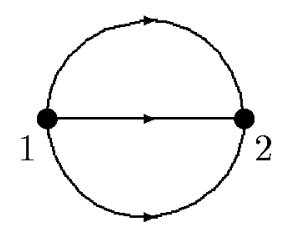

$A_{1}$

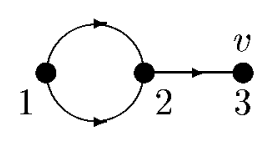

$A_{2}$

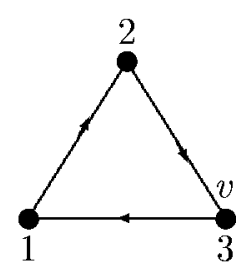

$A_{3}$

FIGURE 1

However, one has

Proposition 3.13. For any choice of a metric $g$ and a metric connection $\theta$ involved in the definition of $\hat{\eta}$,

$$
A_{2}(M)+2 A_{4}(M)=0
$$

if $v=v_{g}$ is the unit volume form determined by the metric.

Proof. First we notice that, by (3.15), we can rewrite

$$
A_{4}(M)=\int_{M} v_{g} P_{g}^{3} v_{g}
$$

By (3.13) we have $d P_{g} v_{g}=0$; since $H^{2}(M)=0$, there exists a form $\gamma \in \Omega^{1}(M)$ such that $P_{g} v_{g}=d \gamma$. By (3.13) we also have

$$
d P_{g}^{2}-P_{g}^{2} d=P_{g} \pi_{h}-\pi_{h} P_{g} .
$$

Therefore, we get

$$
A_{4}(M)=\int_{M} v_{g} P_{g}^{2} d \gamma=\int_{M} v_{g}\left(d P_{g}^{2}-P_{g} \pi_{h}+\pi_{h} P_{g}\right) \gamma=\int_{M} v_{g} P_{g} \gamma
$$

since $\pi_{h} v_{g}=v_{g}$ and $\pi_{h} \gamma=0$. Notice that this expression is independent of the choice of $\gamma$. For, if we take $\gamma^{\prime} \in \Omega^{1}(M)$ such that $d \gamma^{\prime}=P_{g} v$, then $H^{1}(M)=0$ implies $\gamma^{\prime}-\gamma=d \delta$ for some $\delta \in \Omega^{0}(M)$; therefore,

$$
\int_{M} v_{g} P_{g} \gamma^{\prime}-\int_{M} v_{g} P_{g} \gamma=\int_{M} v_{g} P_{g} d \delta=-\int_{M} v_{g} d P_{g} \delta=0 .
$$

Now we introduce the linear operator $R_{g}: \Omega^{*}(M) \rightarrow \Omega^{*+1}(M)$ defined by

$$
R_{g}=\frac{1}{2} \pi_{2 *}\left(\hat{\eta}^{2} \pi_{1}^{*} \alpha\right)
$$


so we can write

$$
A_{2}(M)=2 \int_{M} R_{g} P_{g} v_{g}
$$

Following the same lines of the proof of Prop. 3.4 and using the second identity of (3.2), we can show that

$$
d R_{g}+R_{g} d=-\left(\hat{v}_{g} P_{g}+P_{g} \hat{v}_{g}\right)
$$

where $\hat{v}_{g}$ is the operator that acts by multiplication for the volume form $v_{g}$. Therefore,

$$
A_{2}(M)=2 \int_{M} R_{g} d \gamma=-2 \int_{M}\left(d R_{g}+\hat{v}_{g} P_{g}+P_{g} \hat{v}_{g}\right) \gamma=-2 \int_{M} v_{g} P_{g} \gamma
$$

since $\hat{v}_{g} \gamma=0$. q.e.d.

Remark 3.14. If $\hat{\eta}$ is so chosen as to represent $\bar{P}_{g}=d^{*} \circ G$, then both $A_{2}(M)$ and $A_{4}(M)$ vanish since $\bar{P}_{g} v_{g}=0$.

End of the digression.

3.5. Proof of Theorem 3.9. We will use here a technique similar to that discussed in [2]. That is, we will extend our previous construction from $C_{n}(M)$ to $C_{n}(M) \times I$, where $I$ is a parameter space.

We introduce a parameter $\tau$ ranging over the unit interval $I$, and let all our quantities - the metric $g$, the metric connection $\theta$ and the unit volume form $v$-depend smoothly on $\tau$. Then $A_{\Theta}$ will become a function on $I$. More precisely, we introduce the trivial bundles $C_{n}(M) \times I$ and denote by $\pi$ and $\sigma$ the two projections to $C_{n}(M)$ and $I$ respectively. Then we define

$$
A_{\Theta, \tau}(M)=\sigma_{*}\left(\hat{\eta}_{123}^{3} v_{3}\right)
$$

where now $\hat{\eta}_{123} v_{3}$ is seen as a form in $\Omega^{9}\left(C_{3}(M) \times I\right)$.

As for $v$ we take a representative of the class in $H^{3}(M \times I)=$ $H^{3}(M)$ that satisfies $\sigma_{*} v=1$. Notice that, as a form, $v$ belongs to the completion of $\Omega^{3}(M) \otimes \Omega^{0}(I) \oplus \Omega^{2}(M) \otimes \Omega^{1}(I)$.

To let the connection vary on $I$, we consider it as a connection on the pulled-back bundle

$$
\pi^{-1} P \stackrel{p}{\rightarrow} M \times I .
$$


Now we will construct $\eta$ as a closed form in $\Omega^{2}\left(\partial C_{2}(M) \times I\right)$. As before, we can think of the sphere bundle $\partial C_{2}(M) \times I \rightarrow M \times I$ as $\pi^{-1} P \times_{S O(3)} S^{2}$. Consider the commutative diagram:

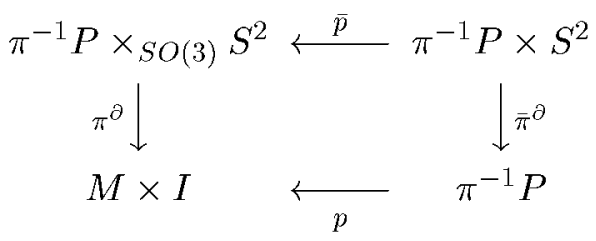

A form $\bar{\eta}$ defined as in (3.1) will be a closed, basic form in $\Omega^{2}\left(\pi^{-1} P \times S^{2}\right)$. As such, it will be the pullback through $\bar{p}$ of a form $\eta \in \Omega^{2}\left(\partial C_{2}(M) \times I\right)$. This form will satisfy the same properties (3.2) and (3.3) described in Prop. 3.1. Moreover, in accordance with Lemma 2.1, we have

Lemma 3.15. If $\eta$ is defined as before, then

$$
\pi_{*}^{\partial} \eta^{3}=\frac{1}{4} p_{1}
$$

where

$$
p_{1}=-\frac{1}{8 \pi^{2}} \operatorname{Tr} F \wedge F=\frac{1}{4 \pi^{2}} F^{i} F_{i}
$$

is the first Pontrjagin form on $M \times I$.

Proof. Consider $\bar{\eta}$ as in (3.1). Since $\omega^{2}=0$, we have

$$
\begin{aligned}
(4 \pi)^{3} \bar{\pi}_{*}^{\partial} \bar{\eta}^{3} & =\bar{\pi}_{*}^{\partial}\left\{3 \omega\left[d\left(\theta^{i} x_{i}\right)\right]^{2}+\left[d\left(\theta^{i} x_{i}\right)\right]^{3}\right\} \\
& =3 d \theta^{i} d \theta^{j} \bar{\pi}_{*}^{\partial}\left(\omega x_{i} x_{j}\right)-3 d \theta^{i} \theta^{j} \theta^{k} \bar{\pi}_{*}^{\partial}\left(x_{i} d x_{j} d x_{k}\right) .
\end{aligned}
$$

A simple evaluation of these integrals shows that

$$
\bar{\pi}_{*}^{\partial}\left(\omega x_{i} x_{j}\right)=\frac{4}{3} \pi \delta_{i j}, \quad \bar{\pi}_{*}^{\partial}\left(x_{i} d x_{j} d x_{k}\right)=\frac{4}{3} \pi \epsilon_{i j k}
$$

Therefore,

$$
(4 \pi)^{2} p^{*} \pi_{*}^{\partial} \eta^{3}=(4 \pi)^{2} \bar{\pi}_{*}^{\partial} \bar{\eta}^{3}=d \theta^{i} d \theta_{i}-\epsilon_{i j k} d \theta^{i} \theta^{j} \theta^{k}
$$

which is equal to $F^{i} F_{i}$ by (3.4). (Notice that $\operatorname{Tr} \theta^{4}=0$.) q.e.d.

The extension of $\eta$ to $\hat{\eta} \in \Omega^{2}\left(C_{2}(M) \times I\right)$ and the definition of $\hat{\eta}_{123}$ as a representative of $H^{2}\left(C_{3}(M) \times I\right)=H^{2}\left(C_{3}(M)\right)$ proceed as before, by taking an appropriate $\rho \in \Omega^{0}\left(C_{2}(M) \times I\right)$ and $\alpha \in \Omega^{2}(M \times M \times I)$. (Notice only that the involution $T$ and the projections $\pi_{i}$ and $\pi_{i j}$ act as the identity on $I$.) In particular, the properties (3.17), (3.19) and (3.20) still hold.

Now we are in a position to define $A_{\Theta, \tau}$ and to prove 
Lemma 3.16. For an arbitrary dependence of $g, \theta$ and $v$ on $I$, we have

$$
A_{\Theta, 1}(M)-A_{\Theta, 0}(M)=\int_{I} d A_{\Theta, \tau}(M)=-\frac{1}{4} \int_{M \times I} p_{1} .
$$

Then Theorem 3.9 follows immediately since

$$
\mathrm{CS}_{1}(M, f)-\mathrm{CS}_{0}(M, f)=\int_{M \times I} p_{1}
$$

Proof. We use formula (3.16) and get

$$
d A_{\Theta, \tau}(M)=-\sigma_{*} d\left(\hat{\eta}_{123}^{3} v_{3}\right)+\sigma_{*}^{\partial} \iota_{\partial}^{*}\left(\hat{\eta}_{123}^{3} v_{3}\right)=\int_{\partial C_{3}(M)} \iota_{\partial}^{*}\left(\hat{\eta}_{123}^{3} v_{3}\right)
$$

since $\hat{\eta}_{123}^{3} v_{3}$ is closed.

We will first consider the principal faces of $\partial C_{3}(M)$, i.e., the faces (12), (23) and (31). The last two are immediately seen to give no contribution since, by (3.20b) and (3.20c), there are no forms depending on the point 1 in the first case and no forms depending on the point 2 in the second case. Therefore, we are left only with the contribution of face (12), viz.,

$$
-\int_{(12)} \eta_{12}^{3} v_{3}=-\int_{\partial C_{2}(M)} \eta^{3}=-\frac{1}{4} p_{1}
$$

by Lemma 3.15 .

To end our proof we have only to show that the integral over the hidden face (123) vanishes. This face is a bundle over $M \times I$,

$$
(123) \stackrel{\pi^{(123)}}{\longrightarrow} M \times I
$$

whose fiber $F$ can be described as $C_{3}\left(\mathbb{R}^{3}\right)$ modulo translations and scalings. Therefore, $F$ is a 5 -dimensional space whose boundary has three components, denoted by $((12) 3),(1(23))$ and $((31) 2)$. A component of $\partial F$, say $((12) 3)$, can be then described as follows: fix the translations by $x_{3}=0$; so $F$ close to a component of the boundary looks like $C_{2}\left(\mathbb{R}^{3}\right)$ divided by scalings. Since $\partial C_{2}\left(\mathbb{R}^{3}\right)$ is a $S^{2}$-bundle over $\mathbb{R}^{3}$, dividing by the scaling makes each component of $\partial F$ a $S^{2}$-bundle over $S^{2}$.

The integral that we want to evaluate can be written as

$$
-\int_{(123)}\left(\eta_{12}+\eta_{23}+\eta_{31}\right)^{3} v_{3}=\int_{M} v \pi_{*}^{(123)}\left(\eta_{12}+\eta_{23}+\eta_{31}\right)^{3} .
$$


We now consider the commutative diagram:

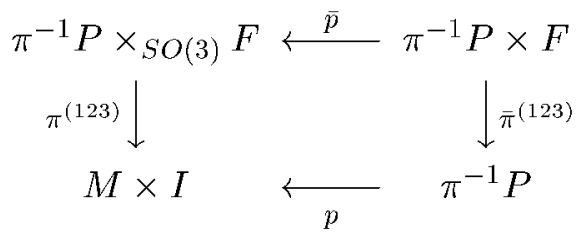

Then, denoting by $\eta_{i j}, \omega_{i j}$ and $x_{i j, k}$ the pullbacks of $\eta, \omega$ and $x_{k}$ through the map $\pi_{i j}$ defined in (3.21), we have

$$
\begin{aligned}
(4 \pi)^{3} p^{*} \pi_{*}^{(123)} & \left(\eta_{12}+\eta_{23}+\eta_{31}\right)^{3} \\
= & (4 \pi)^{3} \bar{\pi}_{*}^{(123)} \bar{p}^{*}\left(\eta_{12}+\eta_{23}+\eta_{31}\right)^{3} \\
= & \bar{\pi}_{*}^{(123)}\left\{\omega_{12}+\omega_{23}+\omega_{31}+d\left[\theta^{i}\left(x_{12, i}+x_{23, i}+x_{31, i}\right)\right]\right\}^{3} \\
= & 3 \theta^{i} \int_{F}\left(\omega_{12}+\omega_{23}+\omega_{31}\right)^{2} d\left(x_{12, i}+x_{23, i}+x_{31, i}\right) \\
= & 3 \theta^{i} \int_{\partial F}\left(\omega_{12}+\omega_{23}+\omega_{31}\right)^{2}\left(x_{12, i}+x_{23, i}+x_{31, i}\right)=0,
\end{aligned}
$$

The last identity follows from the fact that

$$
\left(\omega_{12}+\omega_{23}+\omega_{31}\right)^{2}=0
$$

on $\partial F$. In fact, on a face, say ((12)3), we have

$$
\omega_{12}+\omega_{23}+\omega_{31}=\omega_{12},
$$

and similarly on the other faces. q.e.d.

This concludes the proof of Theorem 3.9.

3.6. The evaluation of $A_{\Theta}$ on the 3-sphere. We may think of $S^{3}$ as the group $S U(2)$. Then, given an element $h$, we take as unit volume form

$$
v=C \operatorname{Tr}\left(h^{-1} d h\right)^{3}, \quad C=\frac{1}{96 \pi^{2}},
$$

where the trace is taken in the adjoint representation.

In a left and right invariant metric the Levi-Civita connection is given by

$$
\nabla_{X_{i}} X_{j}=\frac{1}{2}\left[X_{i}, X_{j}\right]
$$


on a left invariant basis of vector fields. This implies that the connection form on $P$ when pulled back by a left invariant, orthonormal frame $f_{L}$ is given by

$$
f_{L}^{*} \theta=\frac{1}{2} h^{-1} d h
$$

Consider now the orientation reversing involution

$$
\begin{aligned}
\gamma: S^{3} & \rightarrow S^{3}, \\
h & \mapsto h^{-1},
\end{aligned}
$$

and its lifts to $C_{2}\left(S^{3}\right)$ and $C_{3}\left(S^{3}\right)$. With our choice of $v$, we have

$$
\gamma^{*} v=-v
$$

Moreover, if we denote by $R$ the adjoint representation which corresponds to projecting $S^{3}$ to $S O(3)$, we can write

$$
\gamma^{*} f_{L}^{*} \theta=f_{L}^{*} \theta^{R\left(h^{-1}\right)}=h f_{L}^{*} \theta h^{-1}+h d h^{-1} .
$$

Let us now consider the action of $\gamma$ on $\partial C_{2}\left(S^{3}\right)$. On the base we have the action of $\gamma$ defined before; a point $\mathbf{x} \in S^{2}$ is however sent into $-R(h) \mathbf{x}$. In fact, a point in the tangent bundle is given by $h \exp (\mathbf{x})$, with $\mathbf{x}$ in the Lie algebra. Then

$$
\gamma[h \exp (\mathbf{x})]=\exp (-\mathbf{x}) h^{-1}=h^{-1} h \exp (-\mathbf{x}) h^{-1}
$$

By (3.26), we also have

$$
\gamma^{*} F=R(h) F, \quad \gamma^{*} D \mathbf{x}=-R(h) D \mathbf{x} .
$$

Therefore, using (3.5), we conclude that

$$
\gamma^{*} \eta=-\eta
$$

We can always choose $\rho \in C_{2}(M)$ to be invariant under the action of $\gamma$. Then, from (3.7), we see that

$$
\gamma^{*} d \alpha=-d \alpha .
$$

Thus, up to an exact term, we can choose $\alpha$ to be odd and, finally, obtain

$$
\gamma^{*} \hat{\eta}=-\hat{\eta}
$$


consequently,

$$
\gamma^{*} \hat{\eta}_{123}=-\hat{\eta}_{123}
$$

We know by Theorem 3.9 that the value of $A_{\Theta}$ does not depend on these choices, as long as we do not change our connection $\theta$. Therefore, with this fixed choice of $\theta$, we have

$$
A_{\Theta}\left(S^{3}\right)=0
$$

since we have found an involution, $\gamma$, that reverses the orientation of $C_{3}(M)$ but leaves $\hat{\eta}_{123}^{3} v_{3}$ unchanged. Therefore,

$$
I_{\Theta}\left(S^{3}, f_{L}\right)=\frac{1}{4} \mathrm{CS}\left(S^{3}, f_{L}\right)
$$

Moreover, since

$$
d f_{L}^{*} \theta=-2 f_{L}^{*} \theta^{2}
$$

we get

$$
I_{\Theta}\left(S^{3}, f_{L}\right)=\frac{1}{24 \pi^{2}} \int f_{L}^{*} \operatorname{Tr} \theta^{3}=\frac{1}{2}
$$

If we had instead chosen a right invariant section $f_{R}=h^{-1} f_{L} h$, then

$$
f_{R}^{*} \theta=-\frac{1}{2} d h h^{-1}
$$

and we would have obtained

$$
I_{\Theta}\left(S^{3}, f_{R}\right)=-\frac{1}{2} .
$$

Remark 3.17. The left and right framings are related by the adjoint map from $S^{3}$ to $S O(3)$, and hence it has degree 2. So the corresponding Chern-Simons terms differ by 4 (see Remark 3.10). As the Chern-Simons terms of these two framings are clearly opposite in sign, we could have concluded a priori that $\operatorname{CS}\left(S^{3}, f_{L, R}\right)$ must be \pm 2 .

Note also that the same arguments would have worked for $M=$ $S O(3)$ and would have yielded half the answer for the $\Theta$-invariant. 


\section{The higher invariants}

Our first step is the construction of closed forms on $C_{n}(M)$. To do so, we consider the natural projections

$$
\begin{aligned}
\pi_{i}: C_{n}(M) & \rightarrow M, \\
\pi_{i j}: C_{n}(M) & \rightarrow C_{2}(M), \quad i \neq j,
\end{aligned}
$$

and then pull back the volume form $v \in \Omega^{3}(M)$ and the form $\hat{\eta} \in$ $\Omega^{2}\left(C_{2}(M)\right)$. We will denote them by $v_{i}$ and $\hat{\eta}_{i j}$. The combination $\hat{\eta}_{i j k}$ defined in (3.18) is now a closed form in $\Omega^{2}\left(C_{n}(M)\right)$ for any triple of distinct indices $i j k$. Of course, not all these forms are independent. In particular, we notice that

$$
\hat{\eta}_{i j k}=(-1)^{\sigma} \hat{\eta}_{\sigma(j i k)},
$$

where $\sigma$ is a permutation. Finally, if $n=2 V$, a product of $3 V \hat{\eta}_{i j k}$ 's will be a top form on $C_{n}(M)$, while a product of $3 V \hat{\eta}_{i j k}$ 's and one volume form will be a top form on $C_{n+1}(M)$. It is then natural to consider the relation between these integrals and trivalent graphs. We start with the following definition.

Definition 4.1 (Kontsevich). In our context the simplest way to describe the graph cohomology is as follows. We call a decorated graph a graph with oriented edges and numbered vertices (by convention we start the enumeration by 1 ). We require edges always to connect distinct vertices. If two vertices are connected by exactly one edge, we call that edge regular. Moreover, denoting by $V$ the number of vertices and by $E$ the number of edges, we grade the collection of decorated graphs by

$$
\begin{aligned}
& \operatorname{ord} \Gamma=E-V, \\
& \operatorname{deg} \Gamma=2 E-3 V .
\end{aligned}
$$

The $\Theta$-graph has order 1 and degree 0 . Examples of decorated graphs of order 2 are shown in Figure $2 ; \Gamma_{1}$ and $\Gamma_{2}$ have degree 0 , while $\Gamma^{\prime}$ has degree 1 . Notice that a trivalent graph $\Gamma$ has always degree zero; moreover, its order can be written as

$$
\operatorname{ord} \Gamma=\frac{V}{2} \text {. }
$$

To each decorated trivalent graph $\Gamma$ and 3-manifold $M$ we can associate a number $A_{\Gamma}(M)$ given by

$$
A_{\Gamma}(M) \doteq \int_{C_{n+1}(M)} v_{0} \prod_{(i j) \in \Gamma} \hat{\eta}_{i j 0}
$$



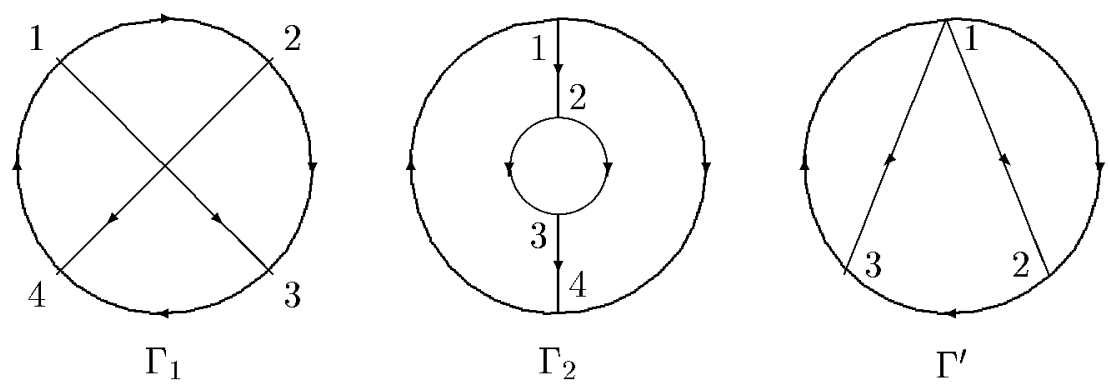

FIGURE 2

where $n=2$ ord $\Gamma$, and $(i j)$ denotes the oriented edge connecting the vertex $i$ to the vertex $j$. Notice that each vertex carries a number between 1 and $n$, while 0 denotes the $(n+1)$-st point in $C_{n+1}(M)$.

Remark 4.2. If we expand the product of $\hat{\eta}_{i j 0}$ 's in $A_{\Gamma}(M)$, the term coming from choosing $\hat{\eta}_{i j}$ in each factor will read

$$
\int_{C_{n+1}(M)} v_{0} \prod_{(i j) \in \Gamma} \hat{\eta}_{i j}=\int_{C_{n}(M)} \prod_{(i j) \in \Gamma} \hat{\eta}_{i j} ;
$$

that is, it corresponds to the usual association of a configuration space integral to a trivalent graph.

Example 4.3. Referring to the trivalent decorated graphs of Figure 2 , we have the following integrals:

$$
\begin{aligned}
& A_{\Gamma_{1}}(M)=\int_{C_{5}(M)} v_{0} \hat{\eta}_{120} \hat{\eta}_{230} \hat{\eta}_{340} \hat{\eta}_{410} \hat{\eta}_{130} \hat{\eta}_{240}, \\
& A_{\Gamma_{2}}(M)=-\int_{C_{5}(M)} v_{0} \hat{\eta}_{140}^{2} \hat{\eta}_{120} \hat{\eta}_{230}^{2} \hat{\eta}_{340}
\end{aligned}
$$

In view of the definition of $A_{\Gamma}(M)$, we give the collection of decorated graphs the structure of an algebra over $\mathbb{Q}$, and extend (4.2) by linearity. We will denote by $\mathcal{D}$ this algebra. Moreover, we introduce the following equivalence relation: if two decorated graphs $\Gamma$ and $\Gamma^{\prime}$ have the same underlying graph, we set

$$
\Gamma=(-1)^{(p+l)} \Gamma^{\prime}
$$

where $p$ is the order of the permutation of the labeling of the vertices to go from $\Gamma$ to $\Gamma^{\prime}$, and $l$ is the number of edges whose orientation must be reversed. Notice that to equivalent graphs we associate the same 
number $A_{\Gamma}(M)$. We will denote by $\mathcal{D}^{\prime}$ the algebra of graphs modulo the above equivalence relation.

Finally, we introduce a coboundary operator $\delta$ on $\mathcal{D}$. By definition, $\delta \Gamma$ is the signed sum of the decorated graphs that are obtained by contracting a regular edge one at a time in $\Gamma$. If the new graph is obtained by contracting the oriented edge connecting the vertex $i$ to the vertex $j$, we relabel the vertices by letting decrease by one the vertices greater than $\max \{i, j\}$ and denote by $\min \{i, j\}$ the vertex where the contraction has happened. Moreover, associate to this contraction a sign $\sigma(i, j)$ defined by

$$
\sigma(i, j)= \begin{cases}(-1)^{j} & \text { if } j>i \\ (-1)^{i+1} & \text { if } j<i\end{cases}
$$

Proposition 4.4. The operator $\delta$ descends to $\mathcal{D}^{\prime}$ and satisfies $\delta^{2}=$ 0 there. Moreover, if we denote by $\mathcal{D}_{n, t}^{\prime}$ the (equivalence classes of) decorated graphs of order $n$ and degree $t$, we have

$$
\delta: \mathcal{D}_{n, t}^{\prime} \rightarrow \mathcal{D}_{n, t+1}^{\prime}
$$

Proof. If we exchange $i$ and $j$ or reverse the arrow connecting them, we get a minus sign from (4.3). In both cases the roles of $i$ and $j$ are exchanged. However, $\sigma(i, j)=-\sigma(j, i)$. Therefore, it does not matter if we exchange $i$ and $j$ first and then apply $\delta$ to the edge $(i j)$ or vice versa.

Then consider three vertices $i, j$ and $k$. We want to prove that the exchange of $j$ with $k$ does not interfere with the action of $\delta$ on $(i j)$. By the previous step, we can assume $i<j$ and $(i j)$ oriented from $i$ to $j$. First suppose $k>i$. We can also assume $k>j$. If we contract $(i j)$ we get a factor $(-1)^{j}$. If we exchange $j$ and $k$ we get a factor -1 and then we have to contract $(i k)$ with a factor $(-1)^{k}$. Now we have to consider what happens to the relabeling. In the first case all indices greater than $j$ are reduced by one, in the second only those greater than $k$. The vertices labeled as $j(j+1) \ldots(k-2)(k-1)$ in the first case are labeled as $(j+1)(j+2) \ldots(k-1) j$ in the second. Since the two strings have length $k-j$ and are related by one cyclic rotation, we get a factor $(-1)^{k-j+1}$ if we want to turn one graph into the other. In summary, if we contract $(i j)$ we get a sign $(-1)^{j}$, while if we echange $j$ and $k$ and then contract $(i k)$ we get a sign $(-1)^{k+1}$ and a labeling that is related 
to the previous one by the sign $(-1)^{k-j+1}$. Similarly, we can treat the case $k<i$. Therefore, $\delta$ descends to $\mathcal{D}^{\prime}$.

To prove that $\delta^{2}=0$ on $\mathcal{D}^{\prime}$, we check that contracting two different pairs $(i j)$ and $(r s)$ in the opposite order gives opposite signs. First assume $j \neq s$ and $i \neq r$. By reordering the vertices, we can assume that $i<j, r<s$ and $j<s$. Then contracting $i$ with $j$ gives $(-1)^{j}$ and let $s$ (and possibly $r$ ) decrease by one, so contracting $r$ with $s$ gives $(-1)^{s-1}$; if we instead contract $r$ with $s$ first and then $i$ with $j$, we get $(-1)^{s}(-1)^{j}$ since $j$ is not reduced by one. If $s=j$ and $i \neq r$, then the two orders in which we can contract the pairs $(i j)$ and $(r j)$ are related by exchanging $i$ with $r$, which changes sign on $\mathcal{D}^{\prime}$. Similarly we treat the case $s \neq j, i=r$.

Finally, we observe that a contraction decreases by one both the number of vertices $V$ and the number of edges $E$ (remember that we contract only regular edges). The last claim is thus a consequence of (4.1). q.e.d.

Example 4.5. The $\Theta$-graph is a cocycle since it has no regular edges.

Example 4.6. Referring to the decorated graphs in Figure 2, we have

$$
\begin{aligned}
& \delta \Gamma_{1}=6 \Gamma^{\prime}, \\
& \delta \Gamma_{2}=2 \Gamma^{\prime} .
\end{aligned}
$$

Therefore, the combination

$$
\Gamma=-\frac{1}{12} \Gamma_{1}+\frac{1}{4} \Gamma_{2}
$$

is a cocycle.

Notice that the action of $\delta$ can be restricted to the algebra of connected graphs. Then we have the following theorem:

Theorem 4.7. If $\Gamma$ is a connected, trivalent cocycle in graph cohomology, then there exists a constant $\phi(\Gamma)$ such that

$$
I_{\Gamma}(M, f)=A_{\Gamma}(M)+\phi(\Gamma) \operatorname{CS}(M, f)
$$

is an invariant for the framed rational homology 3-sphere $M$. Moreover, if ord $\Gamma$ is even, then $\phi(\Gamma)=0$.

From Theorems 3.9, 4.7 and the discussion in subsection 3.6, we get the following corollary: 
Corollary 4.8. If $\Gamma$ is a connected, trivalent cocycle in graph cohomology, then the quantity

$$
J_{\Gamma}(M)=A_{\Gamma}(M)-4 \phi(\Gamma) A_{\Theta}(M)
$$

is an invariant for the rational homology 3-sphere $M$. Moreover, if ord $\Gamma$ is even, then $\phi(\Gamma)=0$; if ord $\Gamma$ is odd, then $J_{\Gamma}\left(S^{3}\right)=0$.

The last statement follows from the fact that, if we choose the connection $\theta$ as in subsection 3.6, then $A_{\Theta}\left(S^{3}\right)=0$; moreover, the involution $\gamma$ we defined there reverses the orientation of $C_{n+1}\left(S^{3}\right)$ since $n$ is even, and sends each $\hat{\eta}_{i j 0}$ and the unit volume form into minus themselves. Since the number of forms $\hat{\eta}_{i j 0}$ is equal to the number of edges of $\Gamma$, that is, to 3 ord $\Gamma$, we see that the integrand is even when ord $\Gamma$ is odd.

4.1. Proof of Theorem 4.7. As in the proof of Theorem 3.9 we introduce the unit interval $I$ on which all our quantities depend. In the following we will use notation similar to that of subsection 3.5. In particular, we still denote by $\pi$ the projection $M \times I \rightarrow M$ and by $\pi^{-1} P$ the pulled-back orthonormal frame bundle.

If $\mathcal{S}$ is a connected component of codimension one of $\partial C_{n}(M) \times I$ describing the coincidence of $p$ points, we will denote by $\pi^{\partial}$ its projection to $C_{n-p+1}(M) \times I$ and by $F$ its fiber. This fiber is given by $C_{p}\left(\mathbb{R}^{3}\right)$ modulo translations and rescalings. Therefore, it is a $(3 p-4)$-manifold with corners. Then we have

Lemma 4.9 (Kontsevich [9]). If $F$ is the fiber of the face $\mathcal{S}$ associated with the collision of $q$ points $\mathbf{x}_{1}, \ldots, \mathbf{x}_{q}$, and by $\omega_{i j}$ we denote the pullbacks of the volume form of the sphere through the projections $\pi_{i j}$ defined in (3.21), then, for any triple of indices $i, j, k(i \neq j, i \neq k)$,

$$
\int_{\mathbf{x}_{i}} \omega_{i j} \omega_{i k}=0
$$

Proof. If $j=k$ the identity follows since $\omega_{i j}^{2}=0$.

If $j \neq k$, then we have at least three vertices in $F$. Thus the integration on $\mathbf{x}_{i}$ extends to all $\mathbb{R}^{3}$ with some points blown up since we can use the other two vertices to fix translations and scalings. The form $\omega_{i j} \omega_{i k}$ is regular except at the points $\mathbf{x}_{i}=\mathbf{x}_{j}$ and $\mathbf{x}_{i}=\mathbf{x}_{k}$; so we can extend the integration of $\mathbf{x}_{i}$ to $\mathbb{R}^{3}$ with only these two points blown up. Next consider the orientation reversing involution

$$
\mathbf{x}_{i}^{\prime}=\mathbf{x}_{j}+\mathbf{x}_{k}-\mathbf{x}_{i}
$$


The forms $\omega_{i j}$ and $\omega_{i k}$ depend only on the difference of $\mathbf{x}_{i}$ with $\mathbf{x}_{j}$ and $\mathbf{x}_{k}$, so they are sent to $\omega_{k i^{\prime}}=-\omega_{i^{\prime} k}$ and $\omega_{j i^{\prime}}=-\omega_{i^{\prime} j}$ respectively. Since this involution reverses the orientation of the manifold and leaves the integrand form unchanged, the integral must vanish. q.e.d.

Let us denote by $\pi_{x}$ the projection $C_{n-p+1}(M) \rightarrow M$ corresponding to the point where all the $p$ points collapsed. Then we define $\tilde{\pi}=\pi \circ \pi_{x}$ and consider the pulled-back bundle $\tilde{\pi}^{-1} P=\pi_{x}^{-1} \pi^{-1} P$. We can now identify $\mathcal{S}$ with $\tilde{\pi}^{-1} P \times_{S O(3)} F$ and consider the commutative diagram:

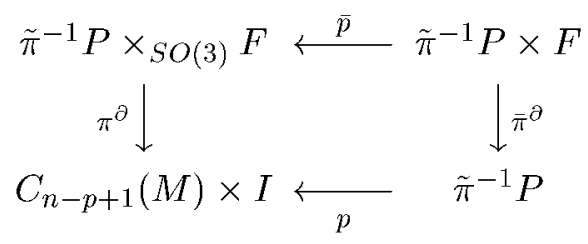

The form $\eta_{i j}$ on this boundary is the pullback of the form $\eta$ on $\partial C_{2}(M) \times$ $I=\pi^{-1} P \times_{S O(3)} S^{2}$ through the projection $\pi_{x, i j}$ given by the composition of the projection $\pi_{i j}: F \rightarrow S^{2}$ defined in (3.21) with the projection $\pi_{x}$. With this notation we can state

Lemma 4.10 (Axelrod and Singer [2]). If $\lambda$ is a form in $\Omega^{*}(\mathcal{S})$ given by a sum of products of forms $\eta_{i j}$, then $\pi_{*}^{\partial} \lambda$ is the pullback through $\pi_{x}$ of a characteristic form on $M \times I$. In particular, it vanishes unless it is of degree zero, in which case it is a constant, or of degree four, in which case it is a multiple of the first Pontrjagin form $p_{1}$.

Proof. By construction $\bar{p}^{*} \lambda$ is a polynomial in $\pi_{x}^{*} \theta$ and $\pi_{x}^{*} d \theta$ with coefficients in $\Omega^{*}(F)$. Therefore, $p^{*} \pi_{*}^{\partial} \lambda=\bar{\pi}_{*}^{\partial} \bar{p}^{*} \lambda$ is a polynomial in $\pi_{x}^{*} \theta$ and $\pi_{x}^{*} d \theta$ and is basic. This means that it is a characteristic form on $C_{n-p+1}(M) \times I$ obtained by pullback from $M \times I$ through $\pi_{x}$. Since $M \times I$ is 4-dimensional, its only characteristic forms are the constant function and the first Pontrjagin form. q.e.d.

Now we introduce the projection $\sigma$ from $C_{n+1}(M) \times I$ to $I$, and, for each decorated trivalent graph, we define

$$
A_{\Gamma, \tau}(M)=\sigma_{*}\left(v_{0} \prod_{(i j) \in \Gamma} \hat{\eta}_{i j 0}\right)
$$

Since the integrand is a closed form, $d A_{\Gamma, \tau}$ will be given only by boundary contributions. We have the following: 
Lemma 4.11. The only boundary terms that contribute to $d A_{\Gamma, \tau}$ come from:

1. faces corresponding to the collapse of two points connected by exactly one edge in $\Gamma$;

2. the face corresponding to the collapse of all points but the point labeled 0; in this case, the contribution is a multiple of the first Pontrjagin form if ord $\Gamma$ is odd, and vanishes if ord $\Gamma$ is even.

Assuming this lemma for the moment, suppose that 1 and 2 are points as in case 1 . Then, on the face where 1 and 2 come together, $\hat{\eta}_{120}=-\eta_{12}$ and its pushforward gives -1 . Notice that the rest of the graph is left unchanged. Now compare what happens if we contract the pair $(i j)$, assuming $i<j$, instead of (12). To do so we bring the pair $i$ to 1 and $j$ to 2 by using cyclic rotations, then contract and rotate again to bring the vertex $i$ into its original position. More explicitly, first we rotate the points $12 \ldots i \ldots j \ldots n$ to $i(i+1) \ldots j \ldots n 12 \ldots(i-1)$. Since we have done $i-1$ cyclic rotations in an even chain, we get the sign $(-1)^{i-1}$. Then we rotate $(i+1) \ldots j$ to $j(i+1) \ldots(j-1)$. This gives $\operatorname{sign}(-1)^{j-i+1}$ since we have done one cyclic rotation in a chain of $j-i$ elements. Then we contract the two vertices. Finally, we rotate $i$ back to its original position; this gives no sign since the chain is odd now. Thus, the contraction of $i$ with $j$, with $i<j$, has sign $(-1)^{j}$. This is in accordance with the sign convention (4.4) in the definition of the coboundary operator $\delta$. Therefore, we have proved

Corollary 4.12. If $\Gamma$ is a connected, trivalent cocycle in graph cohomology, then for an arbitrary dependence of $g, \theta$ and $v$ on $I$,

$$
A_{\Gamma, 1}(M)-A_{\Gamma, 0}(M)=\int_{I} d A_{\Gamma, \tau}(M)=-\phi(\Gamma) \int_{M \times I} p_{1},
$$

where $\phi(\Gamma)$ is a number that depends only on the cocycle $\Gamma$ and vanishes if ord $\Gamma$ is even. More generally, if $\Gamma$ is not a cocycle, we have

$$
d A_{\Gamma, \tau}(M)=-A_{\delta \Gamma, \tau}(M)-\phi(\Gamma) \int_{M \times I} p_{1}
$$

From this corollary we obtain Theorem 4.7.

Proof of Lemma 4.11. To fix our notation, in the following we will denote by $\mathcal{S}$ a boundary face and by $\pi^{\partial}$ its projection to $C_{n-q+2}(M) \times I$, 
where $q(q>1)$ is the number of collapsing points. We will denote by $F$ the $(3 q-4)$-dimensional fiber. We will denote by $\lambda$ the restriction to the boundary of one summand of the integrand form in $A_{\Gamma, \tau}$, and will write

$$
\lambda=\lambda_{1} \pi^{2 *} \lambda_{2}
$$

First we show that the boundary faces involving the point labeled by 0 do not contribute. Suppose that, besides 0 , we have $p$ other points $(p>0$ ) coming together (that is, we are taking $q=p+1$ ). The boundary then projects to $C_{n-p+1}$ and the fiber $F$ has dimension $3 p-1$. Denote by $e$ the number of edges in $\Gamma$ connecting two points on the boundary face $\mathcal{S}$ and by $e_{0}$ the number of edges in $\Gamma$ connecting a point in $\mathcal{S}$ to a point outside $\mathcal{S}$. Since $\Gamma$ is a trivalent graph, we have

$$
2 e+e_{0}=3 p
$$

Notice that, if both $i$ and $j$ belong to $\mathcal{S}$, then $\hat{\eta}_{i j 0}=-\left(\eta_{i j}+\eta_{j 0}+\eta_{0 i}\right)$; if only $i$ belongs to $\mathcal{S}$, then $\hat{\eta}_{i j 0}=-\eta_{i 0}$. Therefore, each edge with at least one vertex in $\mathcal{S}$ contributes with a 2 -form in the vertical direction; that is,

$$
\operatorname{deg} \lambda_{1}=2\left(e+e_{0}\right)=3 p+e_{0}
$$

Since the fiber has dimension $3 p-1$, we see that

$$
\operatorname{deg} \pi_{*}^{\partial} \lambda_{1}=e_{0}+1
$$

Thus, if $e_{0}>0$, then $v_{0} \pi_{*}^{\partial} \lambda_{1}=0$ since $M \times I$ has dimension four. On the other hand, if $e_{0}=0$, then $\pi_{*}^{\partial} \lambda_{1}$ vanishes by Lemma 4.10 .

Next we come to the case where $q$ points $(1<q<n+1)$ come together and the point labeled by 0 is not involved. In this case, if both $i$ and $j$ belong to $\mathcal{S}$, then $\hat{\eta}_{i j 0}=-\eta_{i j}$. If, however, at least one vertex does not belong to $\mathcal{S}$, then $\hat{\eta}_{i j 0}$ will be basic (that is, it will contribute to $\lambda_{2}$ ). Therefore, the form degree in the vertical direction is equal to the number $e$ of edges connecting vertices inside $\mathcal{S}$. Again we have the relation

$$
2 e+e_{0}=3 q
$$

which implies

$$
\operatorname{deg} \lambda_{1}=3 q-e_{0}
$$


Since the fiber has dimension $3 q-4$, we get

$$
\operatorname{deg} \pi_{*}^{\partial} \lambda_{1}=4-e_{0} .
$$

By Lemma 4.10, we see that we have a nonvanishing contribution only if $e_{0}=4$ or $e_{0}=0$; moreover, in the latter case, $\pi_{*}^{\partial} \lambda_{1}$ is a multiple of the first Pontrjagin form. Notice that, since we are considering only connected diagrams, this case corresponds to case 2 in the Lemma we are proving. To prove that this contribution vanishes if ord $\Gamma$ is even, consider the involution that reverses all coordinates $\mathbf{x}_{i}$ in $F$. Since $n$ is even, this involution is orientation reversing. (Notice that we can represent $F$ as $S^{3 n-4}$, with some submanifolds blown up, and that the involution corresponds to the antipodal map.) On the other hand, each $\eta_{i j}$ is sent into $-\eta_{i j}$, and since the number of $\eta$ 's is $E=3$ ord $\Gamma$, we see that the integrand does not change sign if ord $\Gamma$ is even. In this case, then, the integral vanishes.

We are now left with case $e_{0}=4$. Notice that, since $\operatorname{deg} \lambda_{1}=\operatorname{dim} F$ now, we must select the top form on $F$ in $\lambda_{1}$; that is, we must replace each $\eta_{i j}$ with $\omega_{i j} /(4 \pi)$. We have two possibilities:

1. there is at least one vertex in $\mathcal{S}$ connected to a vertex outside $\mathcal{S}$ through exactly one leg;

2. there are two vertices in $\mathcal{S}$ each of which is connected with the outside through exactly two legs (the two legs can connect the vertex inside with the same vertex outside or with two distinct vertices outside), and no other vertex in $\mathcal{S}$ is connected to the outside.

In case 1 , we can apply Lemma 4.9 and conclude that $\pi_{*}^{\partial} \lambda_{1}=0$. In case 2 , we notice that the two vertices under consideration can be connected by an edge only if $q=2$ since the diagram is connected, and this corresponds to case 1 in the Lemma we are proving. Thus, we assume that the two vertices, which we denote by $i$ and $j$, are not connected and $q>2$. In this case, there exists a third vertex $k$ that is conncted to $i$ through exactly one edge to which we associate the form $\omega_{i k}$. Then we integrate $\omega_{i k}$ over the position of the point $i$. If we make the change of variables

$$
\mathbf{x}_{i}^{\prime}=\mathbf{x}_{i}-\mathbf{x}_{k},
$$

we realize that the result of this integration does not depend on $\mathbf{x}_{k}$. Thus, we can see the vertex $k$ as if it were not connected to $i$. It will 
however be connected to two other vertices, possibly not distinct, in $\mathcal{S}$. Then we can use Lemma 4.9 and conclude that $\pi_{*}^{\partial} \lambda_{1}$ vanishes also in this case. q.e.d.

This concludes the proof of Theorem 4.7.

\section{Knots in a rational homology 3 -sphere}

The forms $\hat{\eta}_{i j k}$ we have introduced in the previous sections allow for the construction of invariants of knots $K \subset M$-when $M$ is a rational homology 3 -sphere generalizing the case of knots in $\mathbb{R}^{3}$ discussed in [5].

In general (for details see [5]), an imbedding

$$
f: X \hookrightarrow Y
$$

gives rise to natural imbeddings

$$
f^{n}: X^{n} \hookrightarrow Y^{n}
$$

and

$$
C_{n}^{f}: C_{n}(X) \hookrightarrow C_{n}(Y)
$$

Moreover, since we have natural projections $\pi: C_{n+t}(Y) \rightarrow C_{n}(Y)$, we can consider the pulled-back bundles

$$
\mathcal{C}_{n, t}^{f}=\left(C_{n}^{f}\right)^{-1} C_{n+t}(Y) \rightarrow C_{n}(X) .
$$

We will then have natural projections $\mathcal{C}_{n, t}^{f} \rightarrow \mathcal{C}_{n-r, t-s}^{f}$.

In our case we set $X=S^{1}$ and $Y=M$, and consider a family of imbeddings

$$
K_{\tau}: S^{1} \hookrightarrow M, \quad \tau \in I,
$$

where $I$ is the unit interval, and the correspomding families of imbeddings $C_{n}^{K_{\tau}}$ and of bundles $\mathcal{C}_{n, t}^{K_{\tau}}$.

Notice however that $C_{n}\left(S^{1}\right)$ has $n$ ! distinct connected components. We pick up one of them by choosing a fixed ordering of the points on $S^{1}$. This connected configuration space will be denoted by $\widetilde{C}_{n}\left(S^{1}\right)$. Correspondingly we will have the families of imbeddings $\widetilde{C}_{n}^{K_{\tau}}$ and of bundles $\widetilde{\mathcal{C}}_{n, t}^{K_{\tau}}$. 
Then we recall that we are also interested in varying the metric, the connection form and the unit volume form on $M$. We can take the parameter $\tau$ to belong to the same unit interval $I$, so we are led to consider the map

$$
\begin{aligned}
\hat{K}: S^{1} \times I & \rightarrow M \times I, \\
(\alpha, \tau) & \mapsto \quad\left(K_{\tau}(\alpha), \tau\right),
\end{aligned}
$$

and its generalizations

$$
\hat{\tilde{C}}_{n}^{K}: \widetilde{C}_{n}\left(S^{1}\right) \times I \rightarrow C_{n}(M) \times I .
$$

Finally, the natural projections $\pi: C_{n+t}(M) \times I \rightarrow C_{n}(M) \times I$ allow us to define the pulled-back bundles

$$
\hat{\tilde{\mathcal{C}}}_{n, t}^{K}=\left(\hat{\tilde{C}}_{n}^{K}\right)^{-1}\left(C_{n+t}(M) \times I\right) .
$$

Again we have natural projections $\hat{\tilde{\mathcal{C}}}_{n, t}^{K} \rightarrow \hat{\tilde{\mathcal{C}}}_{n-r, t-s}^{K} ;$ the case $r=n, t=s$ yields the projection

$$
\sigma: \hat{\tilde{\mathcal{C}}}_{n, t}^{K} \rightarrow I
$$

Using the maps $\widetilde{C}_{n}^{K}$ we can pull back the forms $\hat{\eta}_{i j} \in \Omega^{2}\left(C_{n}(M)\right)$. We will keep denoting them by $\hat{\eta}_{i j}$ to avoid cumbersome notation. Similarly we can pull back the forms $\hat{\eta}_{i j} \in \Omega^{2}\left(C_{n}(M) \times I\right)$ by the maps $\hat{\tilde{C}}_{n}^{K}$.

Notice that a form $\hat{\eta}_{i j} \in \Omega^{2}\left(\hat{\tilde{C}}_{n, t}^{K}\right)$ (more precisely we should write $\hat{\tilde{C}}_{n}^{K *} \hat{\eta}_{i j}$ ) depends on $\tau$ in two ways: through the metric connection $\theta$ and through the map $\hat{\tilde{C}}_{n}^{K}$.

As in the case of manifold invariants, we will look for configuration space integrals that yield functions on $I$. A constant function will then be a knot invariant.

The simplest quantity we can write down with these ingredients is the self-linking number

$$
\operatorname{sln}(K, M) \doteq \int_{\widetilde{\mathcal{C}}_{2,0}^{K}} \hat{\eta},
$$

which is not a knot invariant. In fact, given a family of imbeddings $K_{\tau}$, we can write

$$
\operatorname{sln}\left(K_{\tau}, M\right)=\sigma_{*} \hat{\eta}
$$


where $\sigma$ is the projection $\hat{\tilde{C}}_{2,0}^{K} \rightarrow I$. We then note that $\hat{\tilde{C}}_{2}^{K *} v_{i}=0$, so $\hat{\eta}$ is a closed form on $\hat{\tilde{\mathcal{C}}}_{2,0}^{K}$; moreover, $\partial \widetilde{\mathcal{C}}_{2,0}^{K}=S^{1} \times\{-1,1\}$. Therefore, we have

$$
d \operatorname{sln}\left(K_{\tau}, M\right)=\sigma_{*}^{\partial} \hat{\eta}=\int_{\partial \tilde{\mathcal{C}}_{2,0}^{K_{\tau}}} \hat{\eta}=-2 \int_{S^{1}} \psi_{K_{\tau}}^{*} \eta
$$

For a given imbedding $K$, the map

$$
\psi_{K}: S^{1} \rightarrow \partial C_{2}(M)
$$

is defined as follows: Consider the tangent map $K_{*}: T S^{1} \rightarrow T M$ and its restriction to the sphere bundles. Since $S\left(T S^{1}\right)=S^{1} \times\{-1,1\}$, we actually have two maps opposite to each other from $S^{1}$ to $S(T M)=$ $\partial C_{2}(M)$, one corresponding to the point -1 and the other to the point 1. We take $\psi_{K}$ as the latter.

In the next subsection we will show how to associate knot invariants to cocycles in a new graph cohomology. We will see that the only possible failure for the integrals we write down to be true invariants is given by a term proportional to $d \sin \left(K_{\tau}, M\right)$. Therefore, subtracting the correct multiple of the self-linking number, we obtain knot invariants.

5.1. Knot invariants. To define knot invariants, we have to introduce an appropriate graph cohomology. Essentially we use the same diagrams considered in sec. 4 , but with some important modifications.

Definition 5.1. We call a decorated graph for knots a decorated graph with a distinguished loop (representing the knot) on which we orient all the edges consistently. We call external the vertices on the knot and internal the others. We assume that there are always at least two external vertices. We call internal the edges which do not constitute the knot and external those which do. Following [5], we call connected a decorated graph for knots such that its underlying graph is connected after removing any pair of external edges. (In [1], such a graph is called prime.) Finally, denoting by $E$ the number of internal edges and by $V_{i}$ and $V_{e}$ the number of internal and external vertices, we grade the collection of decorated graphs for knots by

$$
\begin{aligned}
\operatorname{ord} \Gamma & =E-V_{i}, \\
\operatorname{deg} \Gamma & =2 E-3 V_{i}-V_{e} .
\end{aligned}
$$

The $\Theta$-graph can be seen as a connected decorated graph for knots of order 1 and degree 0 if its outer circle is reinterpreted as the knot. 

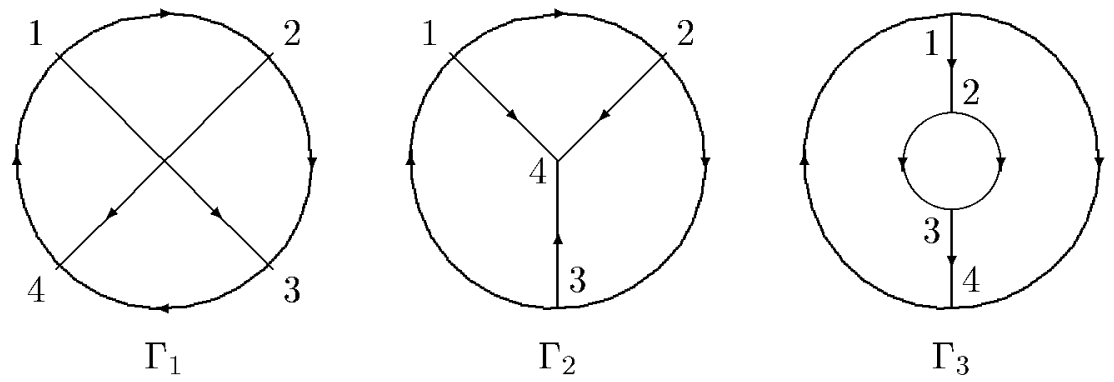

FiguRE 3
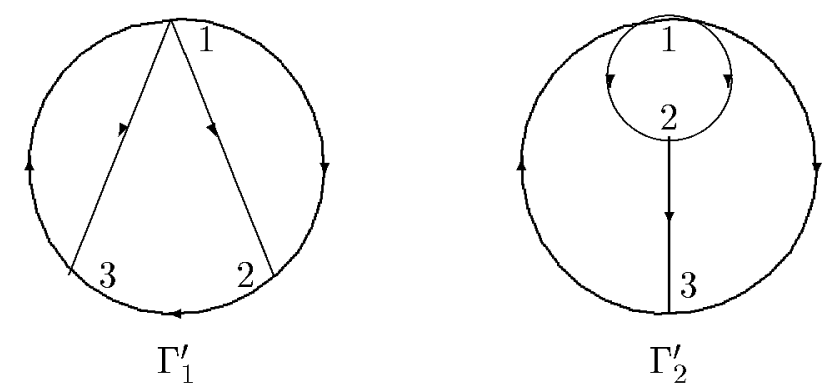

Figure 4

Examples of connected decorated graphs for knots of order 2 and degree 0 are shown in Figure 3; in Figure 3 we have instead connected decorated graphs for knots of order 2 and degree 1 . In all these graphs it is understood that the outer circle represents the knot.

We will follow the convention of labeling first the external vertices following the fixed orientation. Only after we have exhausted the external vertices do we start labeling the internal ones. In this we have the same freedom as we had before, as well as the freedom in orienting the internal edges. We divide the algebra of graphs by the same equivalence relation (4.3) we had before. We keep calling $\mathcal{D}^{\prime}$ the quotient.

To a trivalent graph $\Gamma \in \mathcal{D}^{\prime}$ we can associate the number

$$
A_{\Gamma}(K, M) \doteq \int_{\widetilde{\mathcal{C}}_{n, t+1}^{K}} v_{0} \prod_{(i j) \in \Gamma} \hat{\eta}_{i j 0}
$$

where $n$ and $t$ are the numbers of external and internal vertices in $\Gamma$. Notice that by $(i j)$ now we mean only the internal edges. If we denote by $E$ their number, we have

$$
2 E=n+3 t
$$


since the external vertices are univalent as for the internal edges. This implies that the order of a trivalent graph is 0 and that the integrand in (5.5) is actually a top form on $\widetilde{\mathcal{C}}_{n, t+1}^{K}$. Moreover, in this case we have

$$
\operatorname{ord} \Gamma=\frac{n+t}{2}
$$

In most cases it is possible to replace $\hat{\eta}_{i j 0}$ by $\hat{\eta}_{i j}$ in (5.5). Actually, we have

Lemma 5.2. Unless $(i j)$ belongs to an internal loop, the integral $A_{\Gamma}(K, M)$ in $(5.5)$ does not change if one replaces $\hat{\eta}_{i j 0}$ with $\hat{\eta}_{i j}$.

Proof. Since the dimension at the point 0 is saturated by the volume form $v_{0}$, the integration selects the components of the 2 -form $\hat{\eta}_{i j 0}$ that carry either one form degree at each vertex $i$ and $j$ or two form degrees at one vertex $i$ or $j$ and zero form degrees on the other. The terms $\hat{\eta}_{j 0}$ and $\hat{\eta}_{0 i}$ contribute to the latter case only. Therefore, to prove the lemma, it is enough to show that, unless $(i j)$ belong to an internal loop, integration selects the component of $\hat{\eta}_{i j 0}$ that carries one form degree on $i$ and one on $j$.

Suppose first that $i$ is an external vertex. In this case, it is clear that the integral vanishes by dimensional reasons if we put a zero- or a 2 -form on $i$.

If both $i$ and $j$ are internal we can reason as follows. Suppose we select the component of $\hat{\eta}_{i j 0}$ that carries two form degrees on $i$. Call $j^{\prime}$ and $j^{\prime \prime}$ the other two vertices connected to $i$. Necessarily, the form on one internal edge, say $\hat{\eta}_{i j^{\prime} 0}$, will carry zero form degrees on $i$, while the form on the other internal edge, say $\hat{\eta}_{i j^{\prime \prime} 0}$, will carry one form degree on $i$. Thus, $\hat{\eta}_{i j^{\prime} 0}$ will carry two form degrees on $j^{\prime}$, and so on. Notice that no vertex can appear twice in this sequence since otherwise it would carry a 4 -form. Moreover, no external vertex can belong to the sequence since we cannot put a 2 -form on the knot. Thus, this procedure gives a nonvanishing result only if at some point in this sequence we hit the vertex $j$; in fact, on $j$ we can put a 2 -form since in $\hat{\eta}_{i j 0}$ we have chosen the component that carries no form degrees on $j$. But this can happen only if $(i j)$ belongs to an internal loop. q.e.d.

Example 5.3. Referring to the graphs in Figure 3, and taking into 
account Lemma 5.2, we have the following integrals:

$$
\begin{aligned}
& A_{\Gamma_{1}}(K, M)=\int_{\widetilde{\mathcal{C}}_{4,1}^{K}} v_{0} \hat{\eta}_{130} \hat{\eta}_{240}= \\
& A_{\Gamma_{2}}(K, M)=\int_{\widetilde{\mathcal{C}}_{3,2}^{K}} v_{0} \hat{\eta}_{140} \hat{\eta}_{240} \hat{\eta}_{340}=\hat{\eta}_{13} \hat{\eta}_{24}, \\
& A_{\Gamma_{3}}(K, M)=\int_{\widetilde{\mathcal{C}}_{3,1}^{K}, \hat{\eta}_{14} \hat{\eta}_{24} \hat{\eta}_{34},}, \int_{\widetilde{\mathcal{C}}_{2,3}^{K}} v_{0} \hat{\eta}_{120} \hat{\eta}_{230}^{2} \hat{\eta}_{340}=\int_{\mathcal{\mathcal { C }}_{2,3}^{K}} v_{0} \hat{\eta}_{12} \hat{\eta}_{230}^{2} \hat{\eta}_{34} .
\end{aligned}
$$

On $\mathcal{D}^{\prime}$ we can define a coboundary operator $\delta$ as in the case of decorated graphs for manifolds with the additional constraint that internal edges connecting external vertices are not contracted. Thus, $\delta$ contracts external regular edges or internal regular edges with at least one endpoint internal. Notice that, if the graph has exactly two external vertices, there are no external regular edges. We have then an analogue of Prop. 4.4.

Again we call a cocycle a graph $\Gamma$ killed by $\delta$ and note that $\delta$ can be restricted to the algebra of connected graph.

Example 5.4. The $\Theta$-graph with its outer circle seen as the knot is a cocycle in the graph cohomology for knots since it has no regular edges.

Example 5.5. Referring to the graphs in Figures 3 and 4, we have

$$
\begin{aligned}
& \delta \Gamma_{1}=4 \Gamma_{1}^{\prime}, \\
& \delta \Gamma_{2}=3 \Gamma_{1}^{\prime}+3 \Gamma_{2}^{\prime}, \\
& \delta \Gamma_{3}=2 \Gamma_{2}^{\prime} .
\end{aligned}
$$

Therefore, the combination

$$
\Gamma=\frac{1}{4} \Gamma_{1}-\frac{1}{3} \Gamma_{2}+\frac{1}{2} \Gamma_{3}
$$

is a cocycle in the graph cohomology for knots.

Notice that $\delta \Gamma_{1}$ differs here from what we obtained in Example 4.6 since there we had to contract also the edges (13) and (24).

Finally we can state the following.

Theorem 5.6. If $K$ is a knot in the rational homology 3-sphere $M$ and $\Gamma$ a connected, trivalent cocycle in the graph cohomology for knots, then there exists a constant $\mu(\Gamma)$ such that the quantity

$$
I_{\Gamma}(K, M)=A_{\Gamma}(K, M)+\mu(\Gamma) \sin (K, M)
$$

is a knot invariant. Moreover, $\mu(\Gamma)=0$ if ord $\Gamma$ is even. 
For $M=\mathbb{R}^{3}$, the analogous theorem was proved in [5]; the vanishing of $\mu(\Gamma)$ for ord $\Gamma$ even in this case was proved in [1]. The simplest knot invariant - which corresponds to the cocycle described in Example 5.5 - had previously been described in [8] and [3].

Remark 5.7. In [3] a product structure is introduced on the algebra of graphs. With this product,

$$
A_{\Gamma_{1} \cdot \Gamma_{2}}(K, M)=A_{\Gamma_{1}}(K, M) A_{\Gamma_{2}}(K, M) .
$$

This explains why it is enough to restrict our attention to connected graphs only. (Actually, in [1] it is shown that as for the computation of $\mu(\Gamma)$ it is enough to consider "primitive" graphs, namely, decorated graphs for knots such that their underlying graphs are connected after removing all external edges.)

5.2. Proof of Theorem 5.6. Since the integrand form is closed by construction, then $d I_{\Gamma}$-as a 1 -form on the unit interval $I$-will be given just in terms of boundary integrals.

We first consider the faces corresponding to the collapse of vertices (necessarily internal or carrying the volume form) at an internal vertex. In this case we can use the same arguments we used in subsection 4.1 to prove Theorem 4.7. Essentially we come to the same conclusions of Lemma 4.11, with one important difference: since there are always at least two external vertices and the diagram is connected, it can never happen that all points come together at an internal vertex. Therefore, we are left only with case 1 of Lemma 4.11, and this is taken care of by the action of the coboundary operator $\delta$.

Now consider a collapse at an external vertex. Here both internal and external vertices can come together. Notice that, if the point 0 is involved, the form vanishes since $v$ is a 3 -form and $S^{1} \times I$ is 2-dimensional; so we must only consider the case where $q$ external and $s$ internal vertices (with $q \geq 1, s \geq 0$ and $q+s \geq 2$ ) come together at a point $x$ on the knot. Let us denote by $\mathcal{S}$ the corresponding face. This is a bundle over $I$ whose fiber is a component of $\partial \widetilde{\mathcal{C}}_{n, t+1}^{K_{\tau}}$. However, we can also think of $\mathcal{S}$ as a bundle over $\hat{\tilde{\mathcal{C}}}_{n-q+1, t-s+1}^{K}$ whose projection we denote by $\pi^{\partial}$. Its $(q+3 s-2)$-dimensional fiber can be described as given by $q$ copies of $T_{x} S^{1}$ and $s$ copies of $T_{K_{\tau}(x)} M$ up to global translations along $T_{x} S^{1}$ and scalings, and with the diagonals blown up. Moreover, since we are considering only one connected component of the configuration spaces, we must fix an ordering of the $q$ points on $T_{x} S^{1}$. 
To give an explicit description of this fiber, we consider the following commutative diagram:

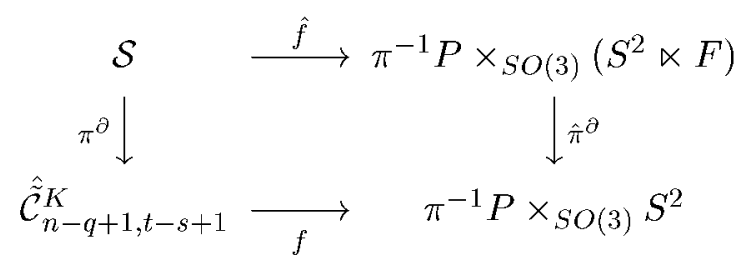

Here $\pi$ is the projection $M \times I \rightarrow M$, and $f$ is the composition of the projection

$$
\pi_{x}: \hat{\tilde{\mathcal{C}}}_{n-q+1, t-s+1}^{K} \rightarrow S^{1} \times I
$$

to the point where the collapse has happened with the map

$$
\begin{array}{rlc}
\hat{\psi}_{K}: S^{1} \times I & \rightarrow & \pi^{-1} P \times_{S O(3)} S^{2} \\
(\alpha, \tau) & \mapsto & \left(\psi_{K_{\tau}}, \tau\right)
\end{array}
$$

with $\psi_{K}$ defined in (5.3). (Remember that $\partial C_{2}(M)=P \times_{S O(3)} S^{2}$.)

The space $S^{2} \ltimes F$ is defined as follows. To each $\mathbf{a} \in S^{2}$ we associate the imbedding

$$
\begin{aligned}
\mathbf{a}: \mathbb{R} & \rightarrow \mathbb{R}^{3}, \\
x & \mapsto \mathbf{a} x .
\end{aligned}
$$

Then we call $F^{\mathbf{a}}$ the configuration space $\widetilde{\mathcal{C}_{q, s}^{\mathbf{a}}}$ modulo translations and scalings; that is, if we denote by $x_{1}, \ldots, x_{q}$ the $q$ coordinates in $\mathbb{R}$ (with $x_{1}<x_{2}<\cdots<x_{q}$ ) and by $\mathbf{x}_{q+1}, \ldots, \mathbf{x}_{q+s}$ the $s$ coordinates in $\mathbb{R}^{3}$, we divide $\widetilde{\mathcal{C}_{q, s}}$ by the translations

$$
\begin{gathered}
x_{i} \rightarrow x_{i}+\xi \\
\mathbf{x}_{j} \rightarrow \mathbf{x}_{j}+\xi \mathbf{a}
\end{gathered}
$$

with $\xi \in \mathbb{R}$, and by the scalings

$$
\begin{gathered}
x_{i} \rightarrow \lambda x_{i}, \\
\mathbf{x}_{j} \rightarrow \lambda \mathbf{x}_{j},
\end{gathered}
$$

with $\lambda \in \mathbb{R}^{*}$. By $S^{2} \ltimes F$ we then mean the pairs $\left(\mathbf{a}, F^{\mathbf{a}}\right)$ with $\mathbf{a} \in S^{2}$.

The action of $S O(3)$ on $S^{2} \ltimes F$ is just the defining action on the copies of $\mathbb{R}^{3}$ and on $S^{2}$, and the trivial action on the copies of $\mathbb{R}$. 
Next consider the form $\lambda \in \Omega^{*}(\mathcal{S})$ given by the restriction to this face of one summand of the integrand form in $I_{\Gamma}$. We can split $\lambda$ as

$$
\lambda=\lambda_{1} \pi^{2 *} \lambda_{2}
$$

Then we have

Lemma 5.8. If $\lambda_{1}$ is a vertical form on $\mathcal{S}$ given by the restriction to this face of a sum of products of forms $\hat{\eta}_{i j}$, then $\pi_{*}^{\partial} \lambda_{1}$ vanishes unless it is a zero-form, in which case it is a constant, or is a 2-form, in which case it is a multiple of $f^{*} \eta$.

Proof. We can write $\lambda_{1}=\hat{f}^{*} \hat{\lambda}_{1}$, where $\hat{\lambda}_{1}$ is a sum of products of pullbacks of the form $\eta \in \pi^{-1} P \times_{S O(3)} S^{2}$ through the projections $\pi_{i j}$ we are going to describe. First define

$$
\pi_{i j}: S^{2} \ltimes F \rightarrow S^{2},
$$

by

$$
\pi_{i j}\left(\mathbf{a}, x_{1}, \ldots, x_{q}, \mathbf{x}_{q+1}, \ldots, \mathbf{x}_{q+s}\right)= \begin{cases}\mathbf{a} \operatorname{sgn}(j-i) & \text { if } i, j \leq q, \\ \rho\left(\mathbf{x}_{j}-\mathbf{a} x_{i}\right) & \text { if } i \leq q, j>q, \\ \rho\left(\mathbf{a} x_{j}-\mathbf{x}_{i}\right) & \text { if } i>q, j \leq q, \\ \rho\left(\mathbf{x}_{j}-\mathbf{x}_{i}\right) & \text { if } i, j>q,\end{cases}
$$

with

$$
\rho(\mathbf{x})=\frac{\mathbf{x}}{|\mathbf{x}|} .
$$

Since the $\pi_{i j}$ 's are equivariant, they descend to

$$
\pi_{i j}: \pi^{-1} P \times_{S O(3)}\left(S^{2} \ltimes F\right) \rightarrow \pi^{-1} P \times_{S O(3)} S^{2} .
$$

Now consider the commutative diagram:

$$
\begin{array}{ccc}
\pi^{-1} P \times_{S O(3)}\left(S^{2} \ltimes F\right) & \stackrel{\bar{p}}{\longleftarrow} \pi^{-1} P \times\left(S^{2} \ltimes F\right) \stackrel{\pi_{i j}}{\longrightarrow} \pi^{-1} P \times S^{2} \\
\hat{\pi}^{\partial} \downarrow & \downarrow \hat{\pi}^{\partial} \\
\pi^{-1} P \times_{S O(3)} S^{2} & \longleftarrow \hat{p} & \pi^{-1} P \times S^{2}
\end{array}
$$

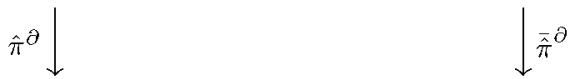

The form $\overline{\hat{p}}^{*} \hat{\lambda}_{1}$ is given in terms of pullbacks of the form $\bar{\eta}=p^{*} \eta$, defined in (3.1), through the projections $\pi_{i j}$. Since the maps $\pi_{i j}$ 's and $\overline{\hat{\pi}}^{\partial}$ act 
as the identity on $\pi^{-1} P$, we conclude that $\overline{\hat{\pi}}_{*}^{\partial} \overline{\hat{p}}^{*} \hat{\lambda}_{1}$ is a polynomial in $\theta$ and $d \theta$ with coefficients in $\Omega^{*}\left(S^{2}\right)$. Moreover, we know that it is basic. Therefore, it must be either a constant or a multiple of the form $\bar{\eta}$. As a consequence, $\hat{\pi}_{*}^{\partial} \hat{\lambda}_{1}$ is either a constant or a multiple of the form $\eta$.

q.e.d.

Now we compute the degree of $\pi_{*}^{\partial} \lambda_{1}$. If $i$ and $j$ are in $\mathcal{S}$, then $\hat{\eta}_{i j 0}$ reduces to $\hat{\eta}_{i j}$. If at least one vertex $i$ or $j$ is not in $\mathcal{S}$, then $\hat{\eta}_{i j 0}$ is basic in $\mathcal{S}$ (that is, it contributes to $\lambda_{2}$ ). Thus, the degree of $\lambda_{1}$ is equal to the number of internal edges connecting vertices inside $\mathcal{S}$. Let us denote this number by $e$, and let $e_{0}$ be the number of internal edges connecting a point in $\mathcal{S}$ with a point outside. Then we have

$$
2 e+e_{0}=q+3 s
$$

since in $\mathcal{S}$ we have $q$ univalent and $s$ trivalent vertices (as for the internal edges). Since the fiber dimension is $q+3 s-2$ we conclude that

$$
\operatorname{deg} \pi_{*}^{\partial} \lambda_{1}=2-e_{0} .
$$

By Lemma 5.8, we then see that $\pi_{*}^{\partial} \lambda_{1}$ vanishes unless $e_{0}=0$ or $e_{0}=2$. In the former instance all points but the point 0 collapse on the knot. The point 0 is now completely disconnected from the point on the knot where all points have collapsed; therefore, we can integrate the volume form on 0 . We are then left with a multiple of $f^{*} \eta=\psi_{K_{\tau}}^{*} \eta$ to be integrated over $\widetilde{\mathcal{C}}_{1,0}^{K}=S^{1}$. But this can also be written as a multiple of $d \sin \left(K_{\tau}, M\right)$ by $(5.2)$.

To prove that we do not have this contribution if ord $\Gamma$ is even, we consider the involution $\phi$ that acts: as the antipodal map on $S^{2}$, trivially on the $q=n$ copies of $\mathbb{R}$, and as the reflection with respect to the origin on the $s=t$ copies of $\mathbb{R}^{3}$. Since the maps $\pi_{i j}$ are equivariant and $\phi^{*} \eta=-\eta$ by Prop. 3.1, we have

$$
\phi^{*} \hat{\lambda}_{1}=(-1)^{E} \hat{\lambda}_{1}
$$

where $E$ is the number of internal edges. The map $\hat{\pi}^{\partial}$ is also equivariant; however, the orientation of the fiber is reversed if $t$ is odd, so

$$
\phi^{*} \hat{\pi}_{*}^{\partial} \hat{\lambda}_{1}=(-1)^{E+t} \hat{\pi}_{*}^{\partial} \hat{\lambda}_{1} .
$$

On the other hand we know that $\hat{\pi}_{*}^{\partial} \hat{\lambda}_{1}$ is proportional to $\eta$, so

$$
\phi^{*} \hat{\pi}_{*}^{\partial} \hat{\lambda}_{1}=-\hat{\pi}_{*}^{\partial} \hat{\lambda}_{1}
$$


This means that $\hat{\pi}_{*}^{\partial} \hat{\lambda}_{1}$ vanishes if $E+t$ is even. By (5.6) and (5.7), this is equivalent to ord $\Gamma$ even.

To complete our proof, we must finally consider the case $e_{0}=2$. In this case the fiber dimension is equal to the degree of $\lambda_{1}$. As in the proof of Lemma 5.8 , we write $\lambda_{1}=f^{*} \hat{\lambda}_{1}$ and see that $\hat{\pi}_{*}^{\partial}$ now selects the part of degree 0 in $\theta$. Therefore, we can use the same arguments used in [5] to prove that $\pi_{*}^{\partial} \lambda_{1}$ vanishes unless $q+s=2$. This case is taken care of by the coboundary operator $\delta$. (Notice that if $\Gamma$ has exactly two external vertices, say 1 and 2 , and we are considering their collapse, then we get two opposite contributions as 1 approaches 2 from one or the other side, provided that 1 and 2 are not connected by one internal edge.)

This concludes the proof of Theorem 5.6.

\section{References}

[1] D. Altschuler \& L. Freidel, Vassiliev knot invariants and Chern-Simons perturbation theory to all orders, Comm. Math. Phys. 187 (1997) 261-287.

[2] S. Axelrod \& I. M. Singer, Chern-Simons perturbation theory, Proc. XXth DGM Conf., (ed. S. Catto and A. Rocha) World Scientific, Singapore, 1992, 3-45; Chern-Simons perturbation theory. II, J. Differential Geom. 39 (1994) 173-213.

[3] D. Bar-Natan, Perturbative aspects of the Chern-Simons field theory, Ph. D. Thesis, Princeton University, 1991; Perturbative Chern-Simons theory, J. Knot Theory Ramifications 4 (1995) 503-548.

[4] R. Bott \& A. S. Cattaneo, Integral invariants of 3-manifolds. II.

[5] R. Bott \& C. Taubes, On the self-linking of knots, J. Math. Phys. 35 (1994) 52475287.

[6] S. S. Chern \& J. Simons, Characteristic forms and geometric invariants, Ann. of Math. 99 (1974) 48-69.

[7] W. Fulton \& R. MacPherson, A compactification of configuration spaces, Ann. of Math. 139 (1994) 183-225.

[8] E. Guadagnini, M. Martellini \& M. Mintchev, Perturbative aspects of Chern-Simons topological quantum field theory, Phys. Lett. B 227 (1989) 111.

[9] M. Kontsevich, Feynman diagrams and low-dimensional topology, First European Congress Math., Paris 1992, Volume II, Prog. Math. 120, Birkhäuser, Basel, $1994,120$. 
[10] C. Taubes, Homology cobordism and the simplest perturbative Chern-Simons 3manifold invariant, Geometry, Topology, and Physics for Raoul Bott, (ed. S.-T. Yau), Internat. Press, Cambridge, 1994, 429-538.

[11] E. Witten, Quantum field theory and the Jones polynomial, Comm. Math. Phys. 121 (1989) 351-399.

HARVARD UNIVERSITY 